\title{
$4<728$ \\ $1+6759$
}

(n)

m.w. 3. SW.

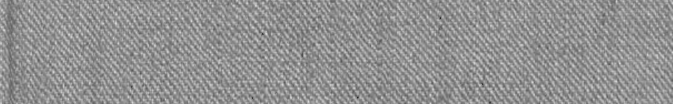

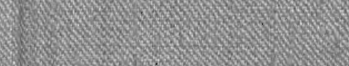

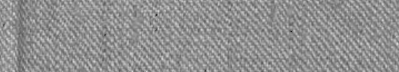


NETS, ETC., USED

\begin{tabular}{l|l|l|l|l|l}
\hline Description & Depth & \multicolumn{2}{|c|}{ TIME } & Speed \\
\hline & & & & & \\
\hline & & & & \\
\hline & & & & & \\
& & & & & \\
\hline $11-5314$ & & & & & \\
\end{tabular}

$$
\text { Psy W } \frac{16.1}{16.2} \cdot D i 7.6 \quad 87 \%
$$

$$
\text { chart 5iod C.4 Y.S. }
$$

Left tangent olouta Catalina sel. bears $X 38^{\circ}-30^{\circ} \mathrm{E}$, dietant $17.2 \mathrm{mi}$
twe 
NETS, ETC., USED

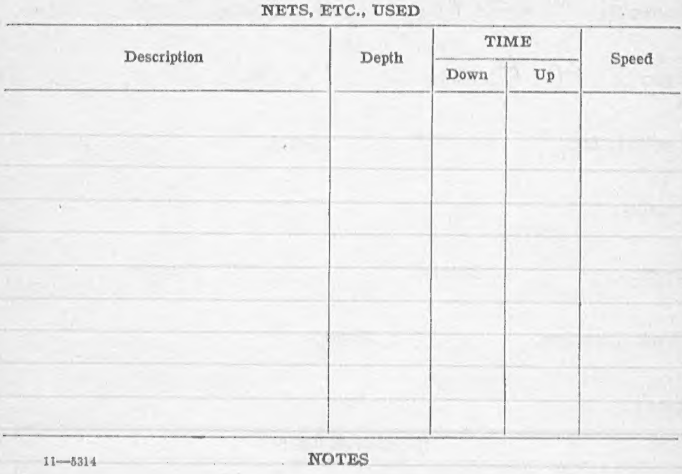

Left thant 5100 C.9 I.S. beass 17 . E diat. $19.7 \mathrm{mi}$ 
Station No. H6730 Date: Gubly 224 1916 Hour: $1: 30$ P.M. to - . . M.

Position: Lat. $33^{\circ}-14^{\prime}-36^{\prime \prime}$ Long. $118-57-00$

Locality:

Depth:

Bottom:

Sample:

Wind: Direction,

Sea:

TEMPERATURES

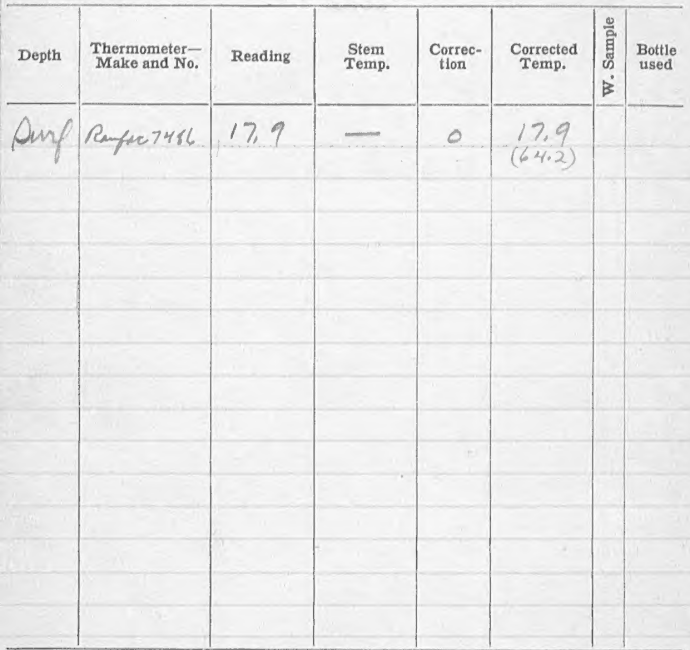

CURRENTS

\begin{tabular}{l|l|l|l|l|l|l}
\hline Depth & Hour & Tide & Time down & Revolutions & Speed & Direction \\
\hline & & & & & \\
\hline & & & & & \\
& & & & & & \\
& & & & & & \\
& & & & & & \\
\end{tabular}

Color of water:

Transparency:

Force,

Sky: 
NETS, ETC., USED

\begin{tabular}{|c|c|c|c|c|}
\hline \multirow{2}{*}{ Description } & \multirow{2}{*}{ Depth } & \multicolumn{2}{|c|}{ TIME } & \multirow{2}{*}{ Speed } \\
\hline & & Down & Up & \\
\hline & & ' & 23 & \\
\hline & & & & \\
\hline & & & & \\
\hline & & & & \\
\hline & & & & \\
\hline & & & & \\
\hline & & & & \\
\hline & & & & \\
\hline & & & & \\
\hline
\end{tabular}

$11-3314$

NOTES

$P_{5 y} \cdot W \times \frac{15}{15.6} \cdot D 17.3 \quad 84 \%$

chant 5200 C.4 Y.S. 
Hour: $200 \rho . M$. to - M.

Position: Lat. $33^{\circ}-14^{\prime}-24^{\prime \prime} \quad$ Long. $119^{\circ}-01^{\prime}-06^{\prime \prime}$

Locality:

Depth:

Bottom:

Sample:

Wind: Direction,

Sea:

Sky:

TEMPERATURES

CURRENTS

\begin{tabular}{l|l|l|l|l|l|l}
\hline Depth & Hour & Tide & Time down & Revolutions & Speed & Direction \\
\hline & & & & & \\
\hline & & & & \\
& & & & & \\
& & & & & \\
& & & & & \\
\hline
\end{tabular}

Color of water:

Transparency: 
NETS, ETC., USED

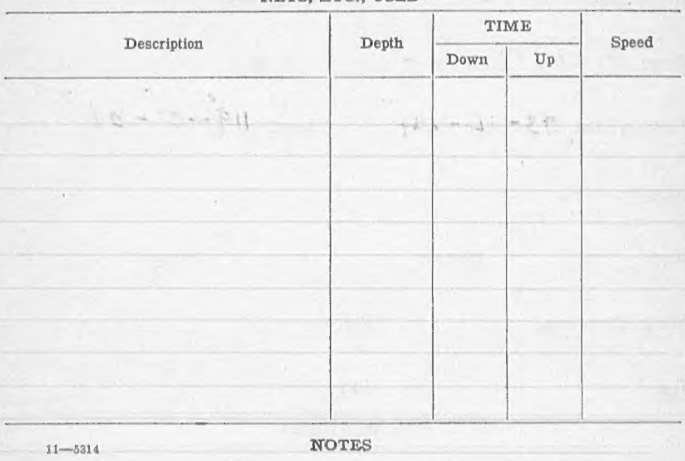

chait $5200 \quad$ C.9 Y.S. 
Station No. 46732

Date: Gul 24

1916
. M.

Long. $119-05-12$

Locality:

Depth:

Bottom:

Sample:

\section{Wind: Direction,}

Sea:

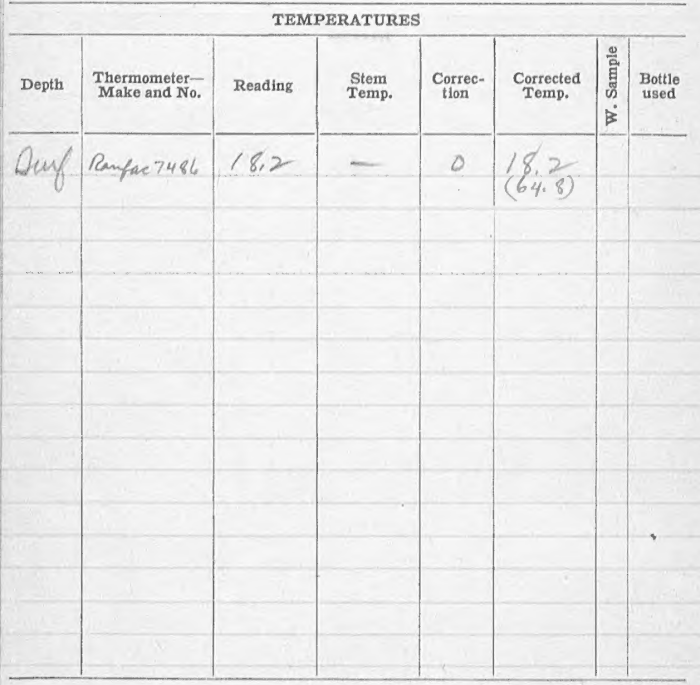

CURRENTS

\begin{tabular}{l|l|l|l|l|l|l}
\hline Depth & Hour & Tide & Time down & Revolutions & Speed & Direction \\
\hline & & & & & & \\
& & & & & & \\
& & & & & & \\
& & & & & & \\
\hline & & & & & \\
\hline
\end{tabular}

REMARKS: Lwale way 
NETS, ETC., USED

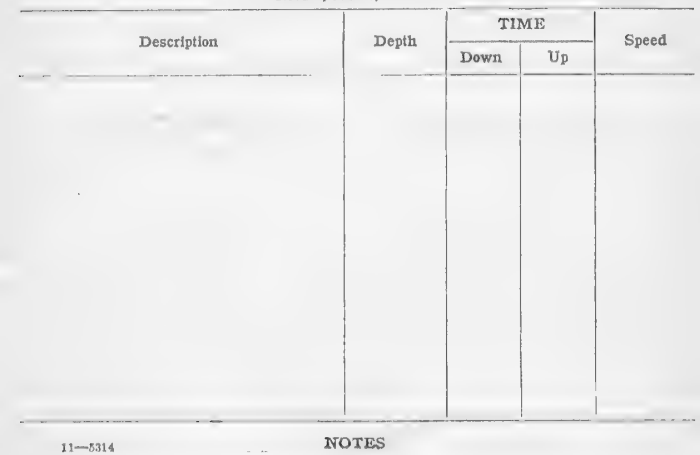

Psy. W $\frac{+5,5}{85,6} D i \geqslant 9$

cirent 5.200 C.7 Y.S. 
Station No.

Hour: $3: 00 \mapsto$. M. to -
Date:

(i, i, ti

191

Position: Lat. $33^{\circ}-13^{\prime}-54^{\prime \prime}$ Long. $119^{\circ}-09^{\prime}-12^{\prime \prime}$

Locality:

Depth:

Bottom:

Sample:

Wind: Direction,

Force,

Sea:

Sky:

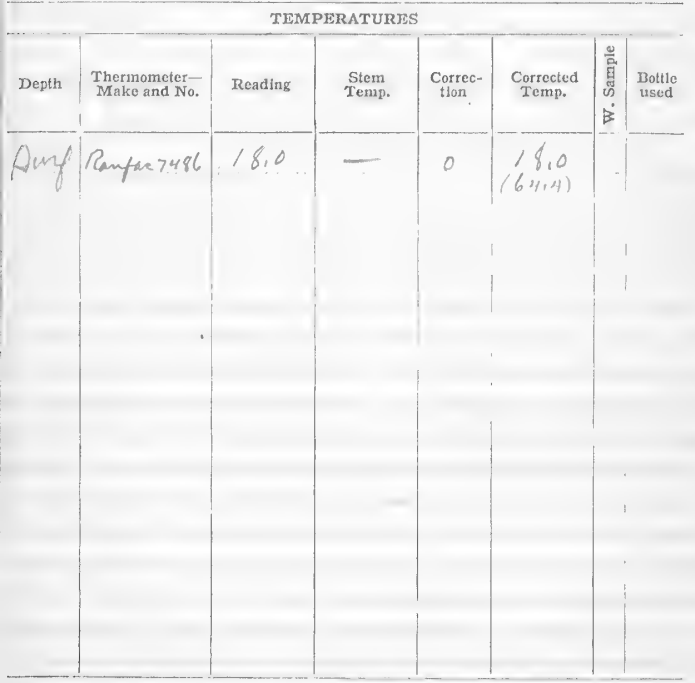

CURRENTS

\begin{tabular}{l|l|l|l|l|l|l}
\hline Depth & Hour & Tide & Time down & Revolutions & Speed & Direction \\
\hline & & & & & & \\
& & & & & & \\
& & & & & & \\
\hline
\end{tabular}

REMARKS: llweGn irmy

$11-5314$ 
NETS, ETC., USED

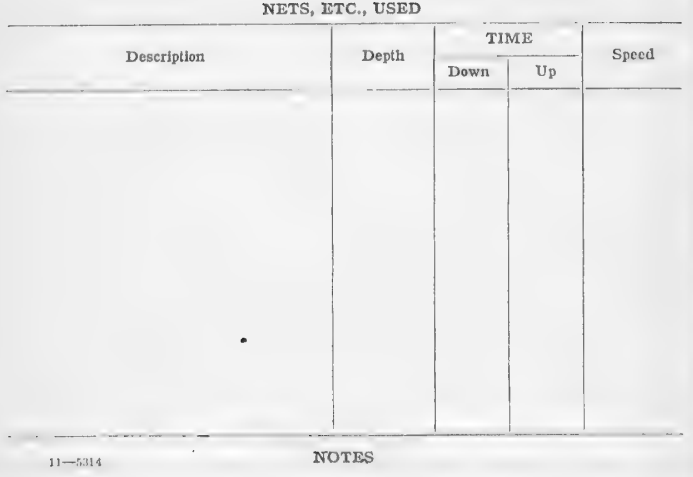

Chout 5200 C.T Y.S. 
Station No. 46734

Hour: $3: 30 \digamma$. M. to -

Position: Lat. $33^{\circ}-13-42^{\prime \prime}$
Date: $9 u h y=4$

${ }_{191} l$

Long. $119^{\circ}-13-06^{\prime \prime}$

Locality:

Depth:

Bottom:

Sample:

Wind: Direction,

Force,

Sea:

Sky:

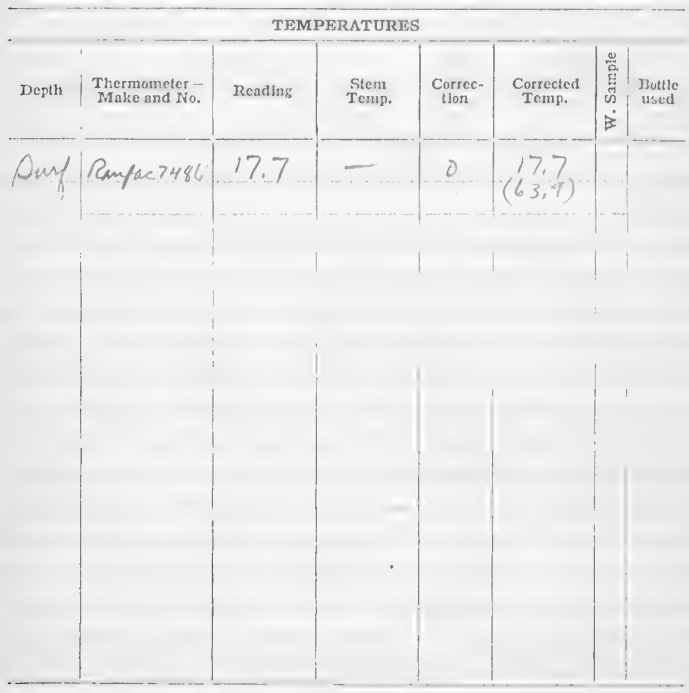

CURRENTS

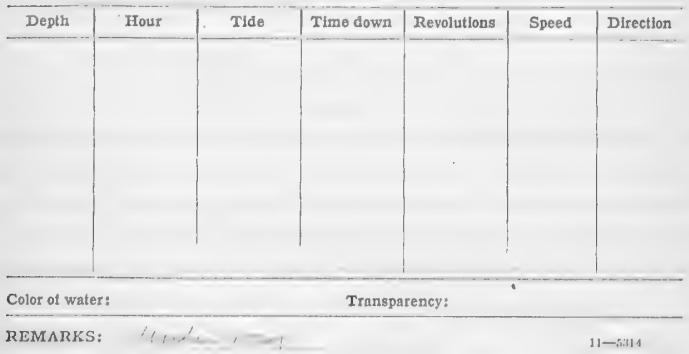


Station No. $/ 1.1 \% 3$

Datc: $, \quad, \cdots$

191

Hour: $4: 00 \quad P$. to $\quad \ldots$ M.

Position: Lat.

Long.

Locality:

Depth:

Bottom:

Sample:

Wind: Direction,

Force,

Sea:

Sky:

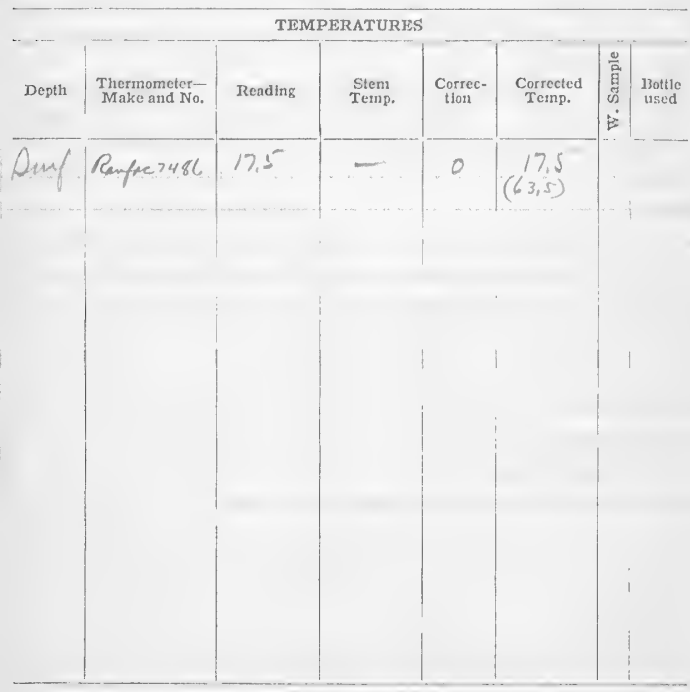

CURRENTS

\begin{tabular}{l|l|l|l|l|l|l}
\hline Depth & Hour & Tide & Time down & Revolutions & Speed & Direction \\
\hline & & & & & \\
& & & & & \\
& & & & & \\
\hline
\end{tabular}

REMARKS;

$11-2314$ 


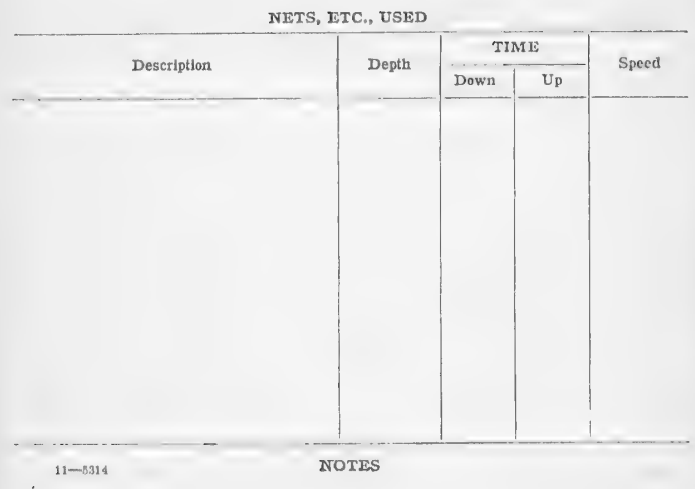

chait 5200 0.7 S.S. Seft tangent San Nicelas shel. hears $5861 / 3 \mathrm{~W}$ (tme) dietant 9.3 miles. 
NETS, ETC., USED

\begin{tabular}{l|l|l|l|l}
\hline Description & Depth & TIME & Speed \\
\hline & Down & Up & \\
\hline & & & & \\
& & & & \\
\end{tabular}

Psy. Wrez $\frac{14.3}{14.3} \quad 015 \% 8 \%$

chast $5200 \quad$ c. 9.5. Left tengent slan Micria da? lieno S 86\% W/tiens) distang 7,3 miles 
Station No. H 6737

Hour: $\sqrt{1}: 00$

P.M. to -

Position: Lat.

\section{Locality:}

Depth:

Bottom:
Wind: Direction,

Sea:

Sky:

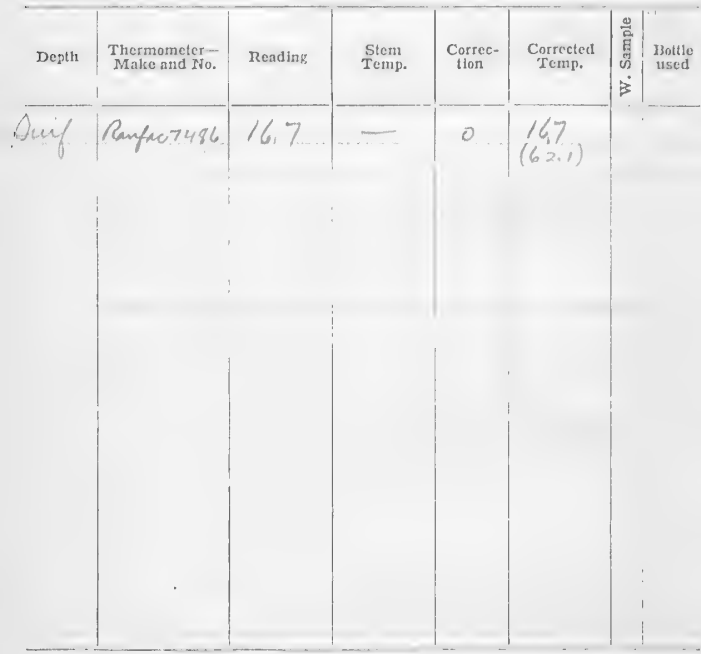

CURRENTS

\begin{tabular}{l|l|l|l|l|l|l}
\hline Depth & Hour & Tide & Time down & Revolutions & Speed & Direction \\
\hline & & & & & \\
& & & & & \\
& & & & & \\
\end{tabular}

Color of water:

Transparency:

REMARKS: hurle way

$11-5314$ 


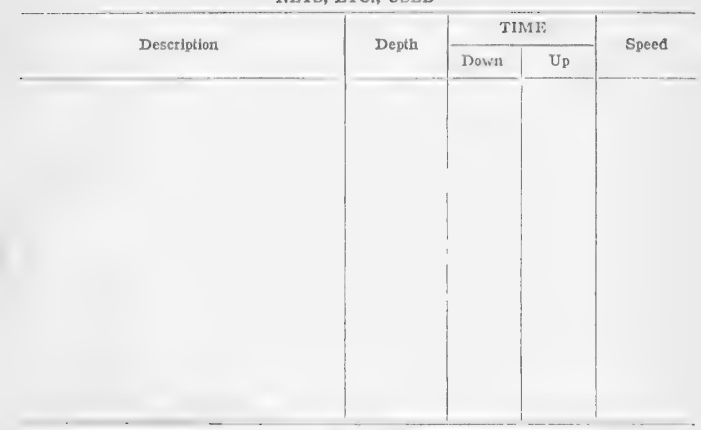

chant 5200 C.4 S.S.

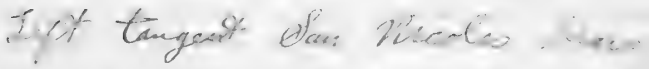
$5861 / 3$ w (two) dewtion at: 


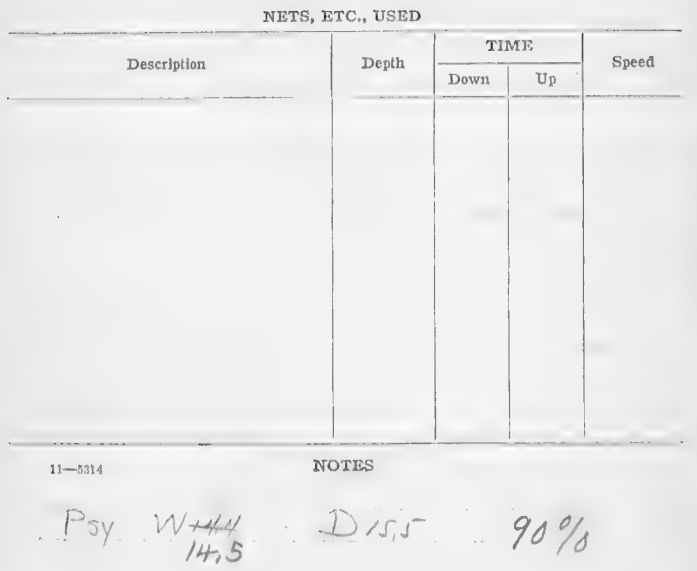

chait 5200 C.F S.S. (anchroge)

Lept tangent elan Niciolins bereso $S 831 / 2$ w (tire) dictent 3.5 miles 
Station No. $14=6 ? ? 0$

Hour: $9: 3^{-1} \cdot \mathbf{M}$. to $10: 15 \div 9 . M$.

Position: Lat.

Long.

Locality: Of Gra Hicons -6
Depth:
Bottom:
Sample:

Wind: Direction, Catm Force, ....
Sea:
G.
Sky:
of $-5-10$

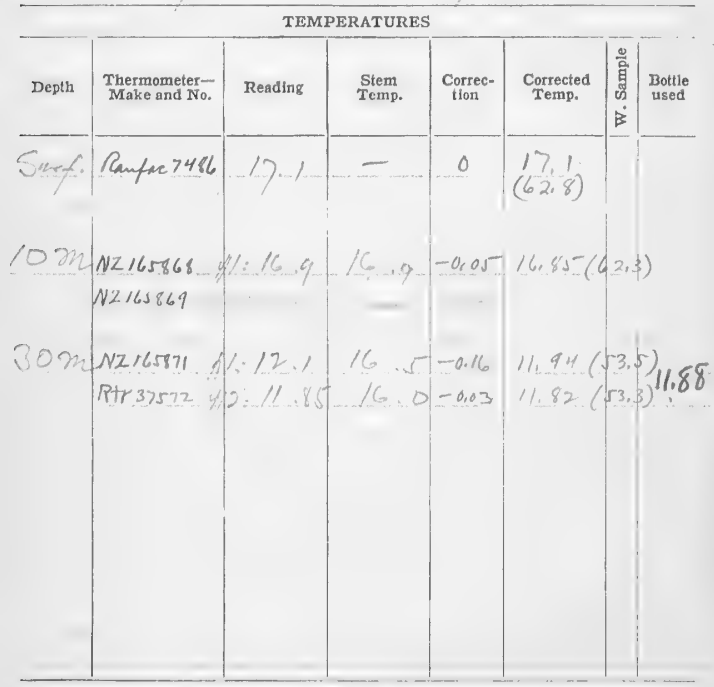

\begin{tabular}{l|l|l|l|l|l}
\hline Hour & Tide & Time down & Revolutions & Speed & Directlon \\
\hline Depth & & & & & \\
\hline & & & & & \\
\hline
\end{tabular}


NETS, ETC., USED

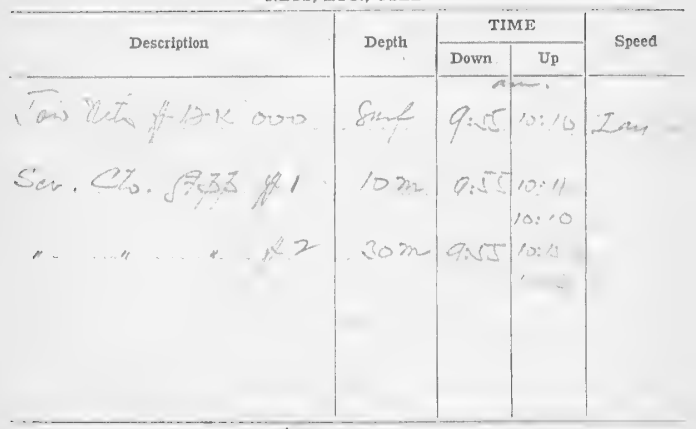

11-5314

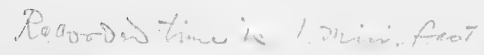

Psy. W $\frac{146}{14.7} D i 7, \quad 77 \%$

Chait 5200 C.T Y.S.

$q \frac{4.5}{4.7 \pi}$. to 12:00 noon

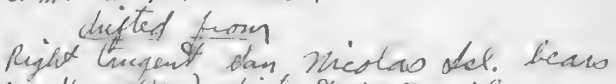
$410 \%$ w (Ime) hidant 4.5 miles Right tangent plan niedine del heavo $n 5 \frac{1}{3}$ in (ture) diatrint 5,0 niti- 
Station No. $4-6 / 39$ Date: $\mathrm{FeC} ; 1916$

Hour: $10: y, 4 . \mathbf{M}$. to $10: 4 \%$ A. M.

Position: Lat.

Long.

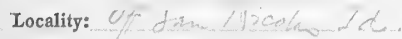
Depth:
Bottom:
Sample:

Wind: Direction,

An ton Force,

Sea: $\quad$ sky: $\quad F-f s-10$

TEMPERATURES

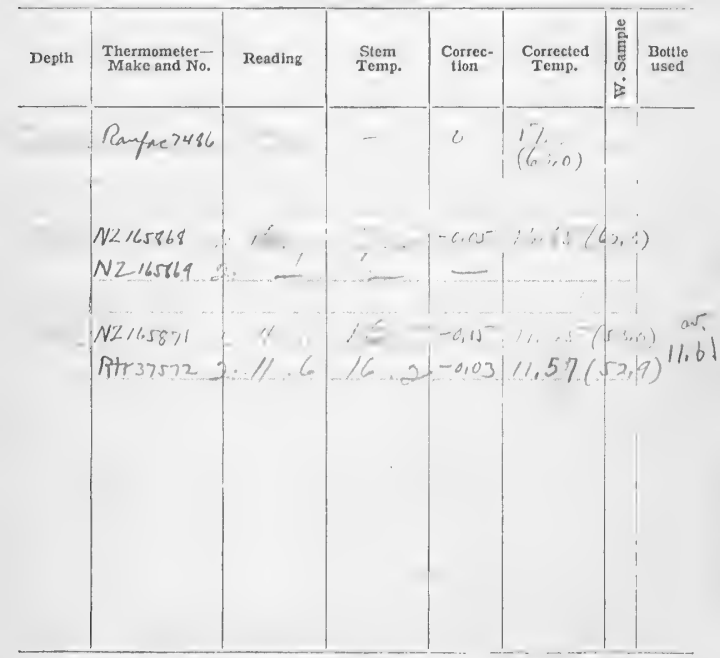

CURRENTS

\begin{tabular}{l|l|l|l|l|l|l|}
\hline Depth & Hour & Tide & Time down & Revolutions & Speed & Direction \\
\hline & & & & & \\
\hline & & & & & \\
& & & & & \\
\end{tabular}

Color of water:

Transparency: 
NETS, ETC., USED

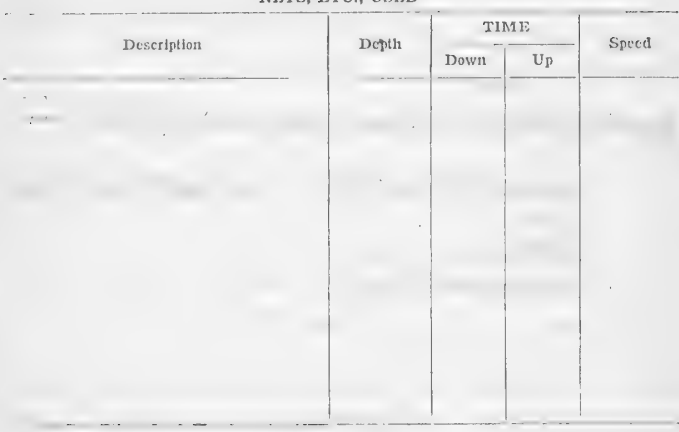


NETS, ITC., USED

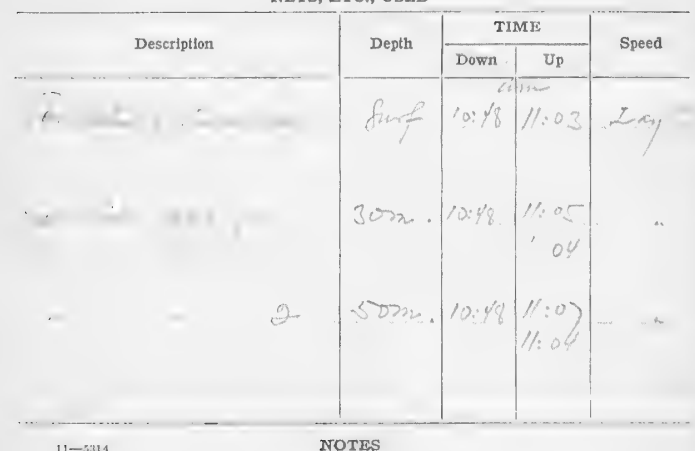

$11-5314$

$\int \operatorname{sen} x+5 \cos x+2+2$ e.

Pesurer tim one mein.

Psy. W $\frac{1.7}{15,0}$

D) 6.6

$84 \%$ 

Hour:
. M. to
. M.

Position: Lat.

Long.

Locality: 0,2

Depth:

Bottom:

Sample:

Wind: Direction, S7r Force, ,

Sea: $\quad \frac{\pi}{7} \quad$ Sky: $\quad 0 \%-75-10$

TEMPERATURES
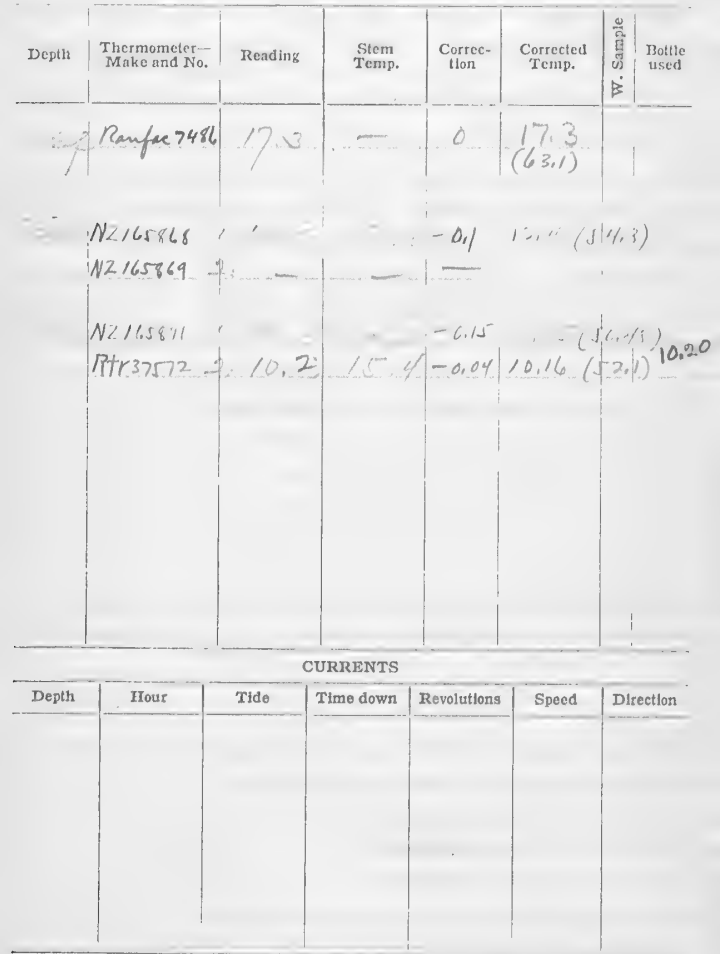

Color of water:

Transparency:

$$
\text { Pisentide. }
$$


NETS, ITC., USED

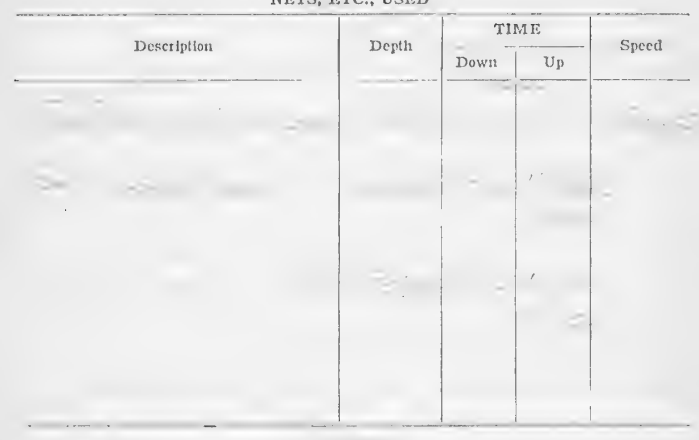


NETS, ETC., USED

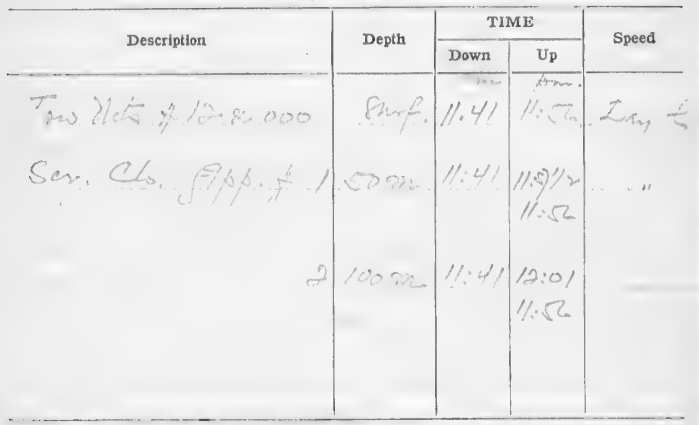

$11-8314$

NOTES

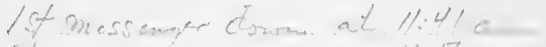

$$
\begin{aligned}
& \text { के . 11: } \sqrt{6} \\
& \text { lleoprited thime is armoct } \\
& P S y_{1} W \underset{15.4}{15.4} \quad D / 8.2 \quad 75 \%
\end{aligned}
$$

Chait 5200 0.\% 5.d.

12:00 hoon to 3:50 P.M.

driftud from

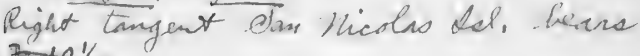
asetey

15\% w distant 5.0 milio (i...)

to

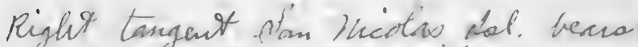
on $23 \mathrm{~W}$ rictiver 7.3 milus

i 7 n...) 
Station No. \& $\because ; \%$

Date: () : $\div$

1916

Hour: $12 i 33$ F. M. to ..12:4f

Position: Lat.

Long.

Locality:

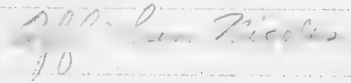

Depth:

Bottom:

Sample:

Wind: Direction,

Force, $0=1$

Sea: $>$

Slry:

TEMPERATURES

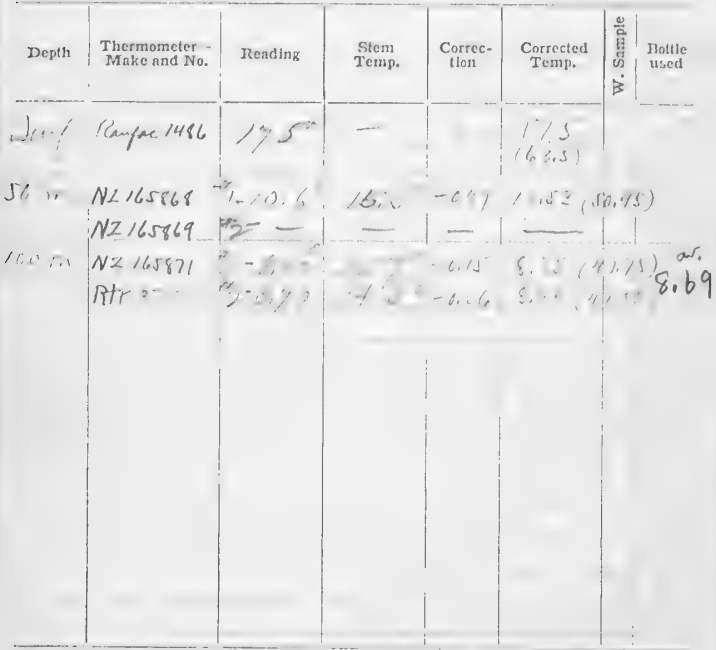

CURRENTS

\begin{tabular}{l|c|c|c|c|c|c}
\hline Depth. Hour & Tide & Time down & Revolutions & Speed & Dlroction \\
\hline & & & & & \\
& & & & & & \\
& & & & &
\end{tabular}

Transparency: 


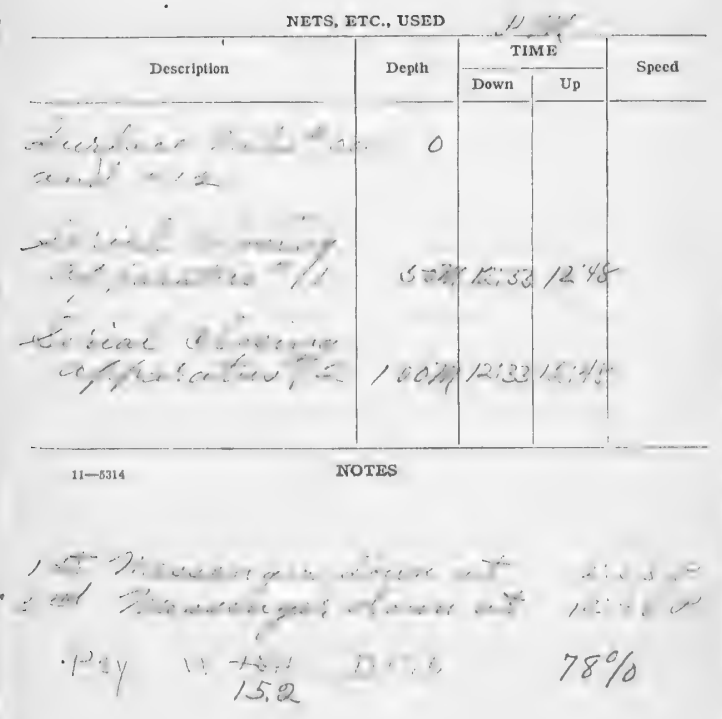


Hour:

. M. to

. M.

Position: Lat.

Long.

Locality:

Depth:

Bottom:

Sample:

Wind: Direction,

Force, $\sigma-1$

Sea:

Sky:

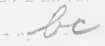

TEMPERATURES

\begin{tabular}{|c|c|c|c|c|c|c|c|}
\hline Depth & $\begin{array}{l}\text { Thermometer- } \\
\text { Make and No. }\end{array}$ & Reading & $\begin{array}{l}\text { Stcm } \\
\text { Temp. }\end{array}$ & $\begin{array}{c}\text { Correc- } \\
\text { tlon }\end{array}$ & $\begin{array}{l}\text { Corrected } \\
\text { Temp. }\end{array}$ & 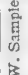 & $\begin{array}{l}\text { Botle } \\
\text { used }\end{array}$ \\
\hline & Router 74 & & - & 0 & $\begin{array}{c}17,6 \\
(6-3,7)\end{array}$ & & \\
\hline
\end{tabular}

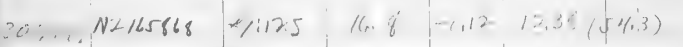

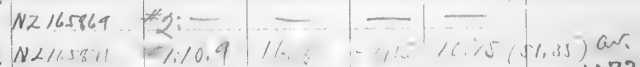

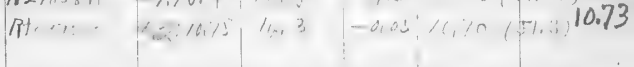
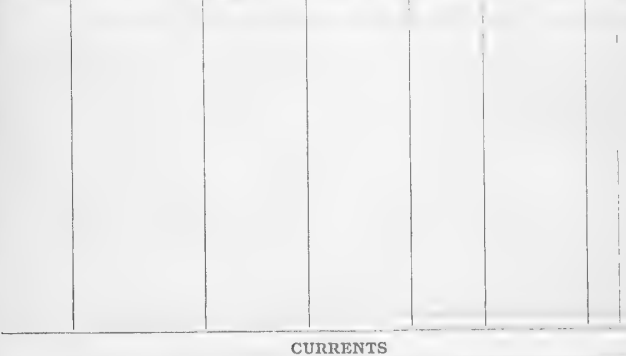

\begin{tabular}{l|l|l|l|l|l|l|}
\hline Depth & Hour & Tide & Time down & Revolutions & Speed & Direction \\
\hline & & & & & & \\
& & & & & & \\
\hline Color of water: & & & & & \\
\hline
\end{tabular}

REMARKS:

$11-\$ 314$ 


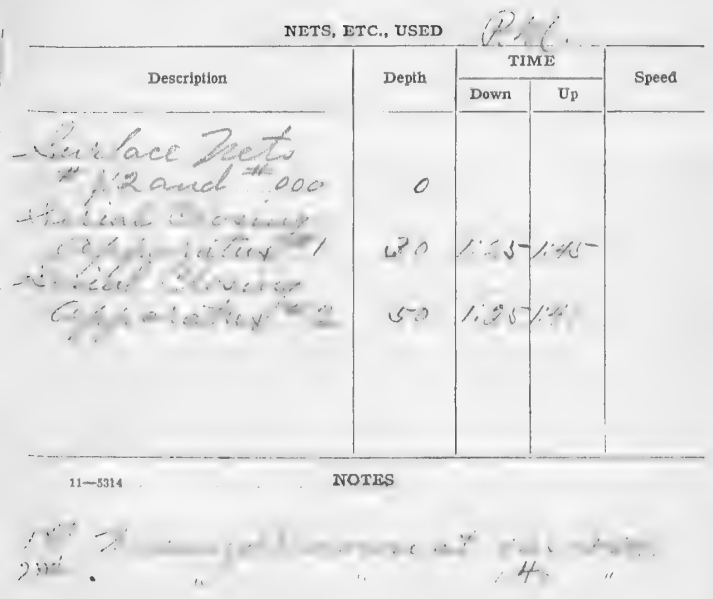


Station No. 6.739

Date: (Lely 2u- $191 \dot{6}$

Hour: listo P.M. to $2 / 1 / \quad P$.M.

Position: Lat.

Long.

Locality:

Depth:

Bottom:

Sample:

Wind: Direction,

Force, $1-2$

Sea: $>1$

Sky: fe

TEMPERATURES

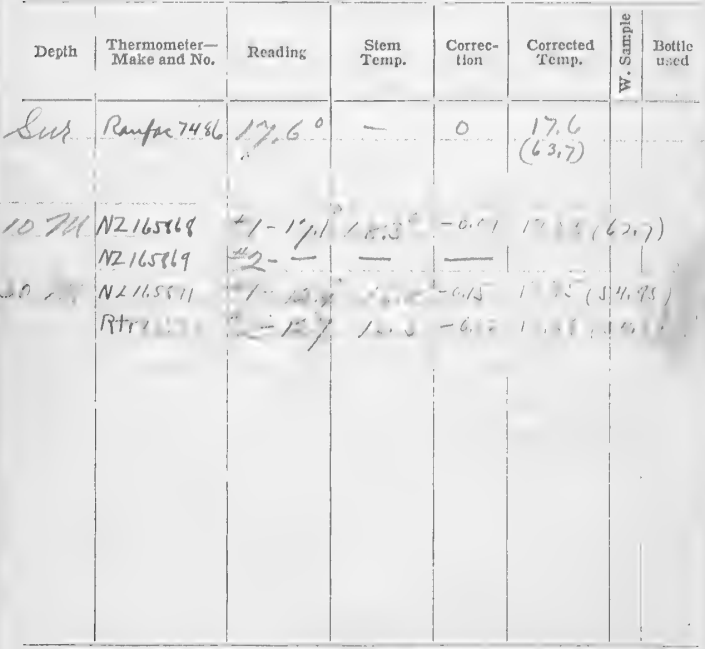

CURIRNTS

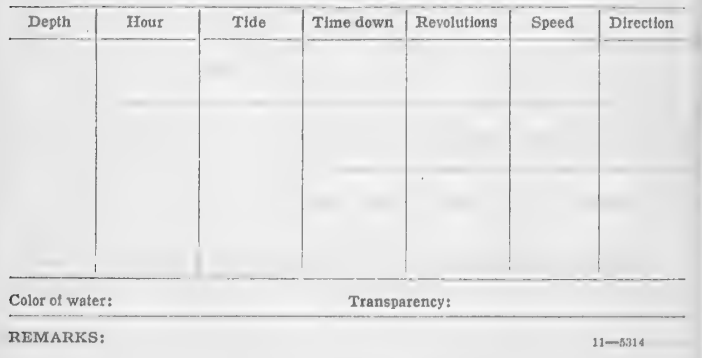


Station No. H.6\%.3\%

Date:

Hour: $;+$. M. to $2 ; 3\} ; \div$.

Position: Lat.

\section{Long.}

Locality:

Depth:

Bottom:

Sample:

Wind: Direction,

Force, $2-3$

Sea:

Sky:

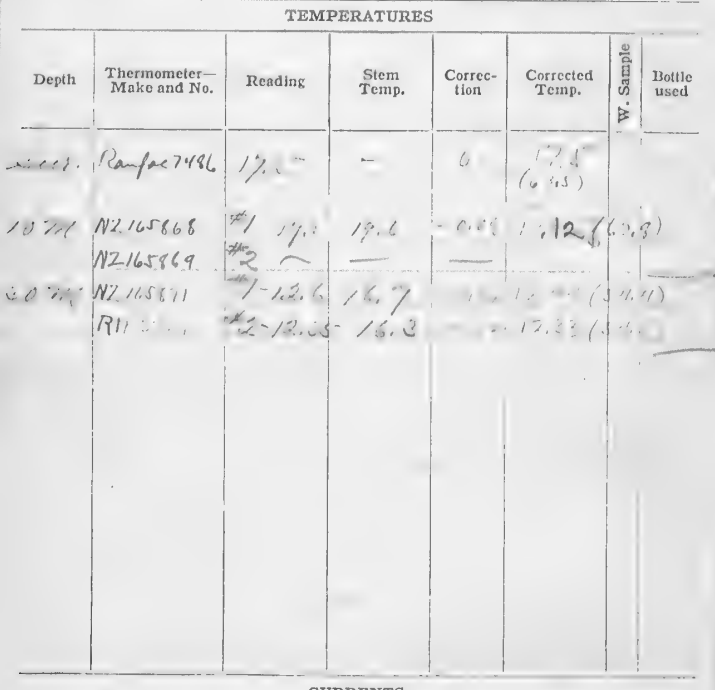

CURRENTS

\begin{tabular}{l|l|l|c|c|c|c|}
\hline Depth & Hour & Tide & Time down & Revolutions & Speed & Direction \\
\hline & & & & & \\
& & & & & \\
& & & & & \\
\end{tabular}

Color of water:

Transparency:

REMARKS: 
NETS, ETC., USED

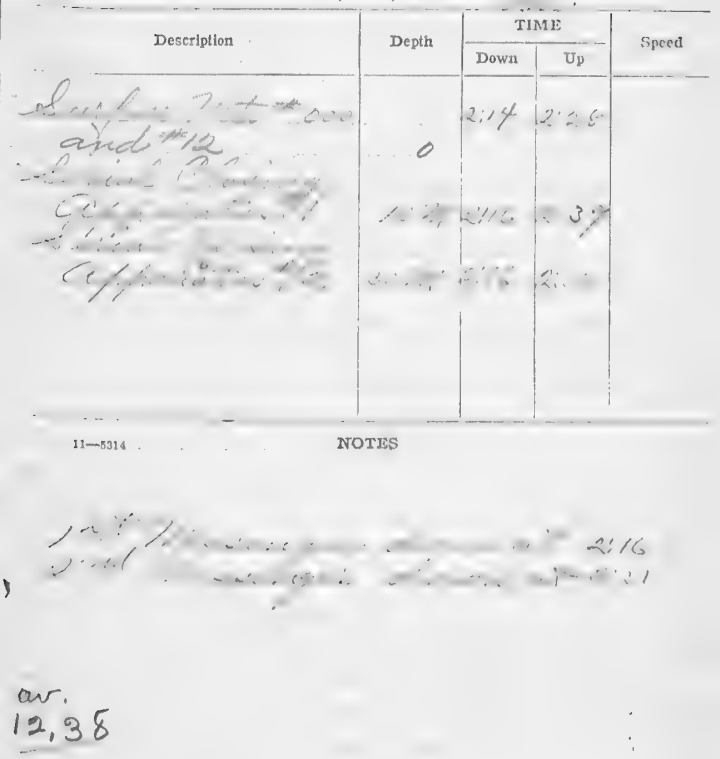


NETS, ETC., USED

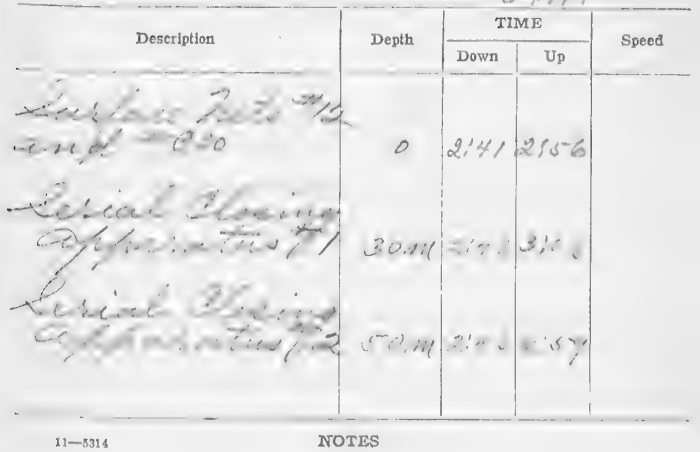

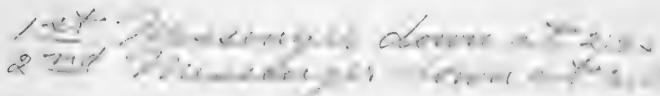
PGy, w $\frac{15,6}{15,7}$
$D, 8,9$
$72 \%$

air

10.50 
Station No. $\&\}$

Date:

Hour: 3:09 P.M. to $3: 3 /$ P. M.

Position: Lat.

Long.

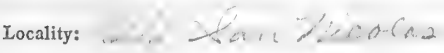

Depth:

Bottom:

Sample:

Wind: Direction, ............ Force, . 2-3

Sea:

Sky: Lfec

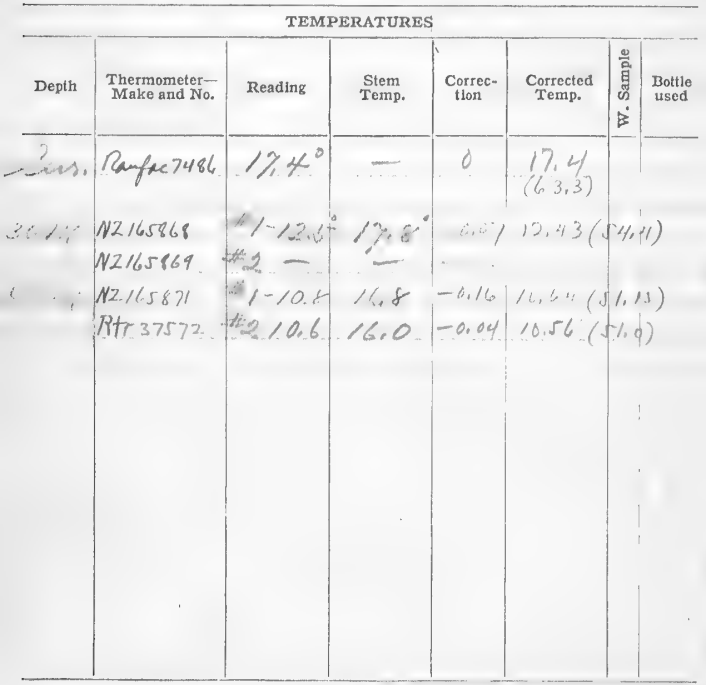

CURRENTS

\begin{tabular}{l|l|l|l|l|l|l}
\hline Depth & Hour & Tide & Time down & Revolutions & Speed & Direction \\
\hline & & & & & \\
\hline & & & & & \\
& & & & & & \\
\hline
\end{tabular}

Transparency: 
NITS, ETC, USED

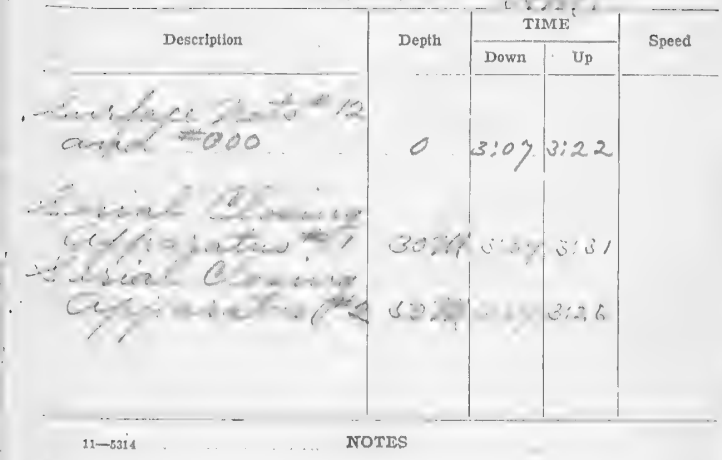

.

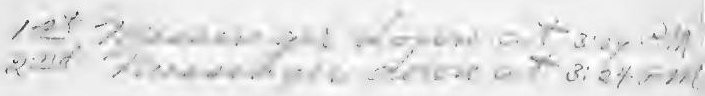

av.

10.60 
Station No. Af 6y3

Hour:

. M. to
Date: .... (incy as is

. $M$.

Position: Lat.

Long.

Locality:

$\because \therefore \operatorname{coc} \cos _{2}$

Depth:

Bottom:

Sample:

Wind: Direction,

Force, $2-13$

Sea: $>4$

Sky:

TEMPERATURES

\begin{tabular}{|c|c|c|c|c|c|c|c|}
\hline Depth & $\begin{array}{l}\text { Thermometer- } \\
\text { Make and No. }\end{array}$ & Reading & $\begin{array}{l}\text { Stem } \\
\text { Temp. }\end{array}$ & $\underset{\substack{\text { Correc- } \\
\text { tion }}}{ }$ & $\begin{array}{l}\text { Corrected } \\
\text { Temp. }\end{array}$ & 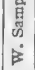 & $\begin{array}{l}\text { Bottle } \\
\text { used }\end{array}$ \\
\hline & Panfec? & 17.4 & - & 0 & $\begin{array}{c}17,4 \\
(63,3)\end{array}$ & & \\
\hline
\end{tabular}

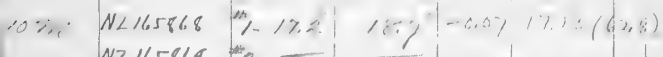

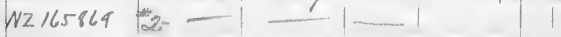

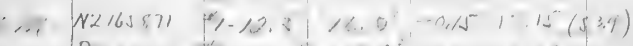

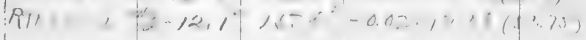

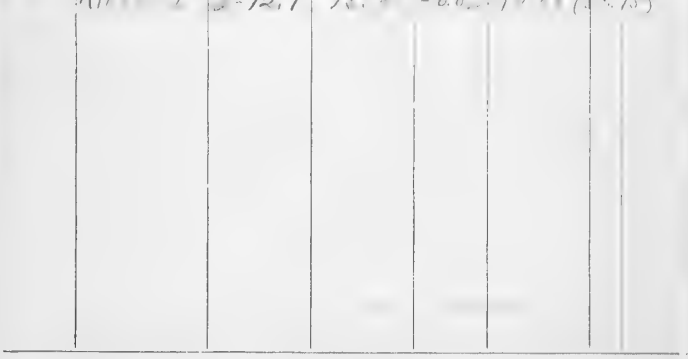

CURRENTS

\begin{tabular}{l|l|l|l|l|l|}
\hline Depth & Tide & Time down & Revolutions & Speed & Direction \\
\hline & & & & & \\
& & & & & \\
& & & & & \\
\end{tabular}

Color of water:

Transparency: 
Description

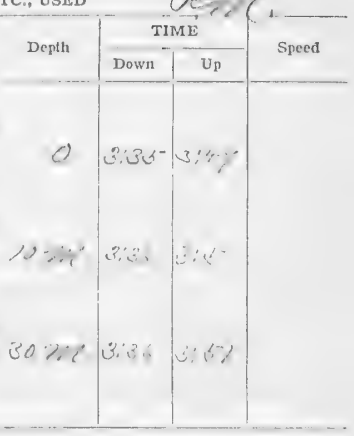

tect,

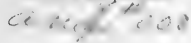

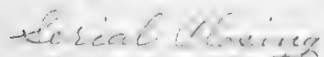

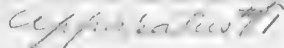

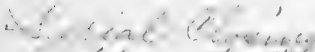

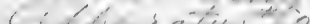

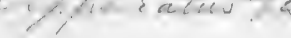

$11-8314$

NOTES

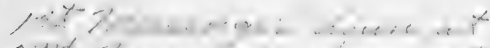

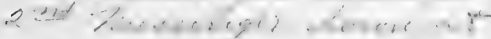

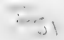

$$
15.1
$$

Eis $2, \ldots$ $3+1+\cdots$

ans

12,12 
Station No. $41-6 ; 40$

Date: \& $1 \mathrm{Ce}, 76$

1916

Hour: $90 ?$ a. м. to $\% 27$ a.

Position: Lat.

Long.

Locality: $\int_{i}$ if $\quad$ is.

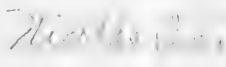

Depth: $S 3-2$ mo Bottom:

Sample:

$$
243 \mathrm{~m}
$$

Wind: Direction,

Force,

Sea:

Sky:

\section{TEMPERATURES}

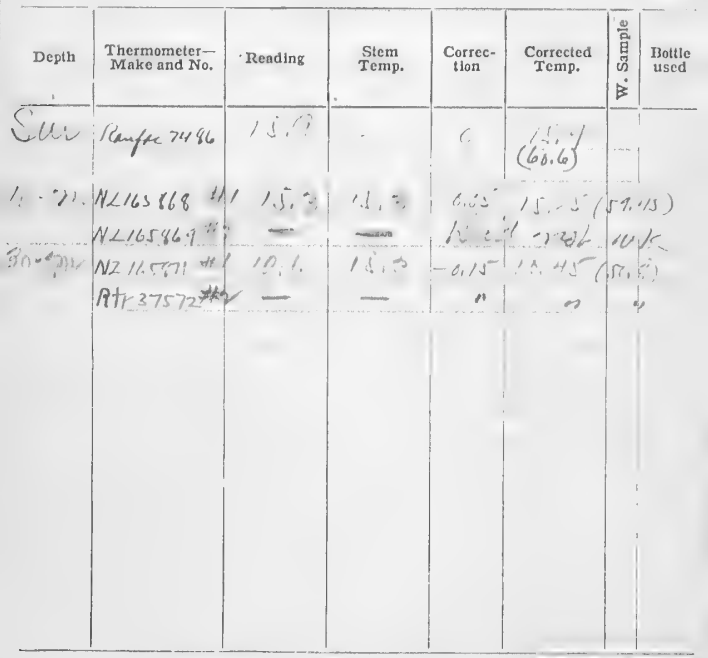

CURRENTS

\begin{tabular}{l|c|c|c|c|c|c}
\hline Depth & Irour & Tide & Time down & Revolutions & Speed & Direction \\
\hline & & & & & \\
& & & & & & \\
& & & & & & \\
\hline
\end{tabular}

Transparency: 
Station No. $\because f-67 L^{\prime}$ Datc: 11\%: 1916 Hour: 73 ?. M. to $95 ;$ M.

Position: Lat. Long.

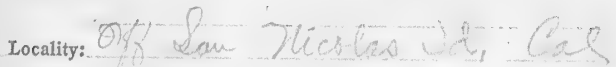

Depth: $53-71$ Bottom:

$$
243 n
$$

Wind: Direction,

Sea:

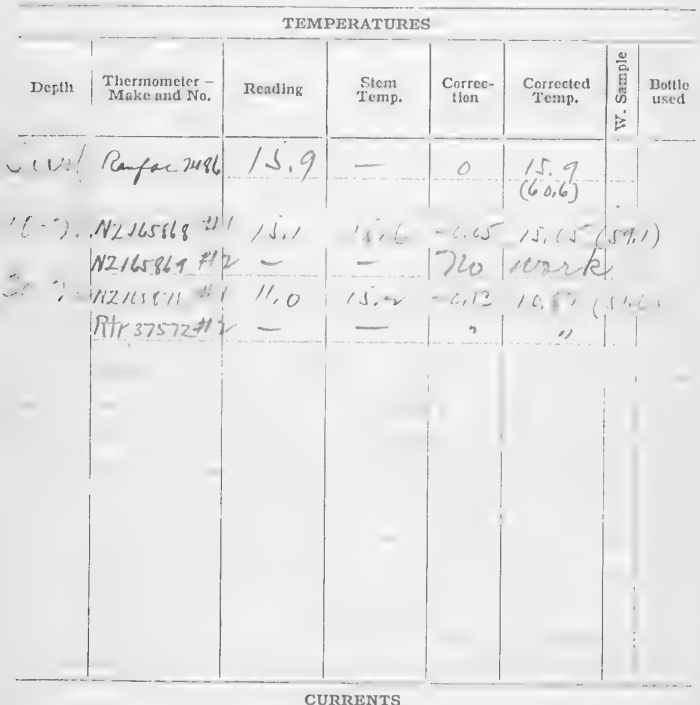

CURRENTS

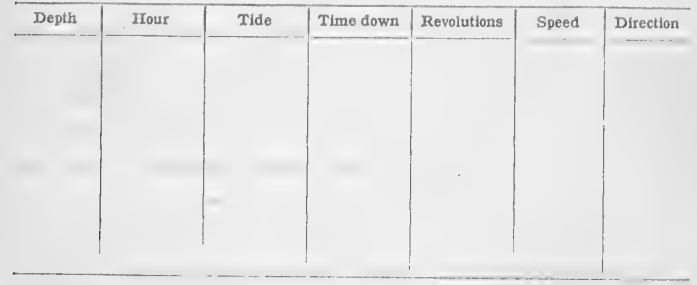

Color of water:

Transparency:

REMARKS:

11- 5314 
NETS, ETC., USED

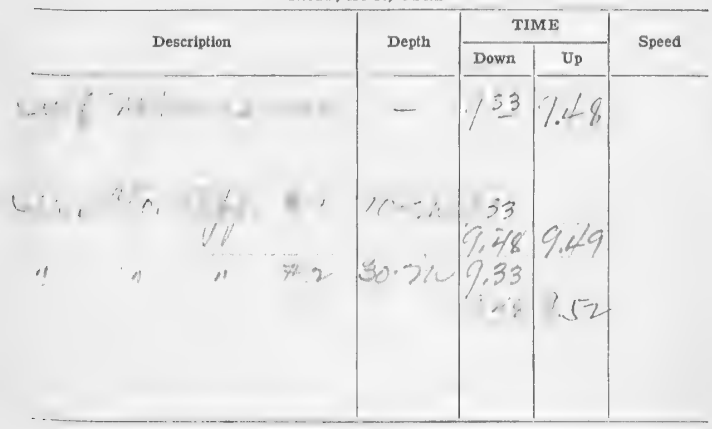

$11-5314$

NOTES

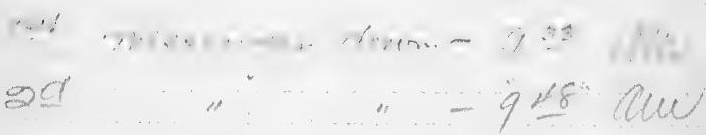

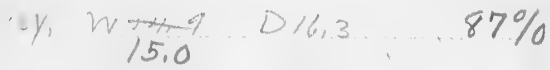


NETS, ETC., USED

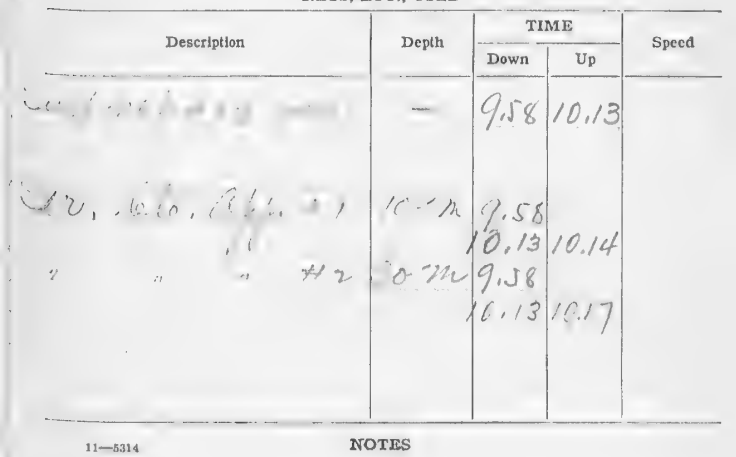

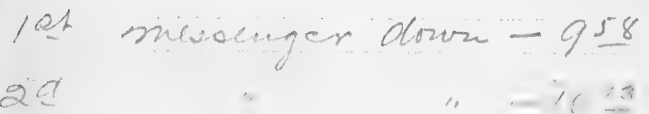

$0,5$.
10.149 
Station No. Date:

IIour: !

Position: Lat.

Long.

Locality: 0 -

Depth: $5-2 W$ Bottom: …… Sample:

Wind: Direction, _....... Force,

Sea: _......... Sky:

TEMPERATURES

\begin{tabular}{|c|c|c|c|c|c|c|c|}
\hline & & & & & & & \\
\hline Depth & $\begin{array}{l}\text { Thermometer- } \\
\text { Make and No. }\end{array}$ & Reading & $\begin{array}{l}\text { Stem } \\
\text { Temp. }\end{array}$ & $\begin{array}{l}\text { Correc- } \\
\text { tion }\end{array}$ & $\begin{array}{l}\text { Corrected } \\
\text { Temp. }\end{array}$ & 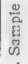 & $\begin{array}{l}\text { Botlle } \\
\text { used }\end{array}$ \\
\hline & anfec 7486 & $:$ & - & & $(6,2,1)$ & & \\
\hline
\end{tabular}

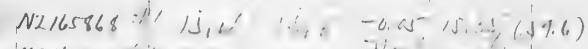
N2165869: 7! -

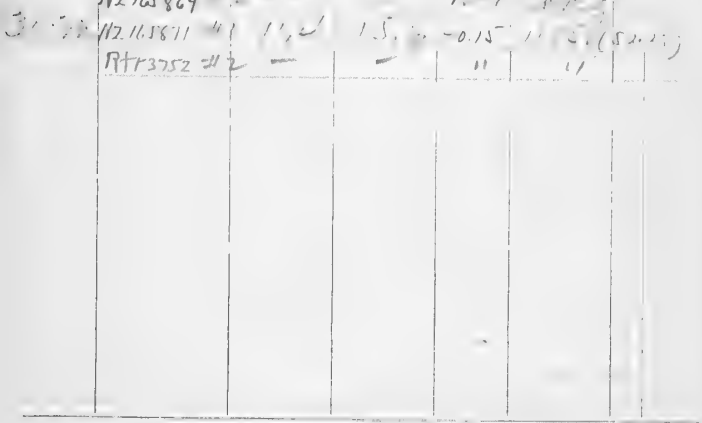

CURRENTS

\begin{tabular}{l|l|l|l|l|l|l}
\hline Depth & Hour & Tlde & Time down & Revolutions & Speed & Direction \\
\hline & & & & & \\
& & & & & & \\
& & & & & & \\
\hline
\end{tabular}

REMARKS:

11-5311

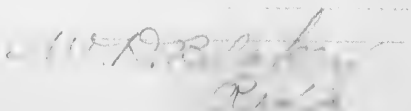


NETS, ETC., USED

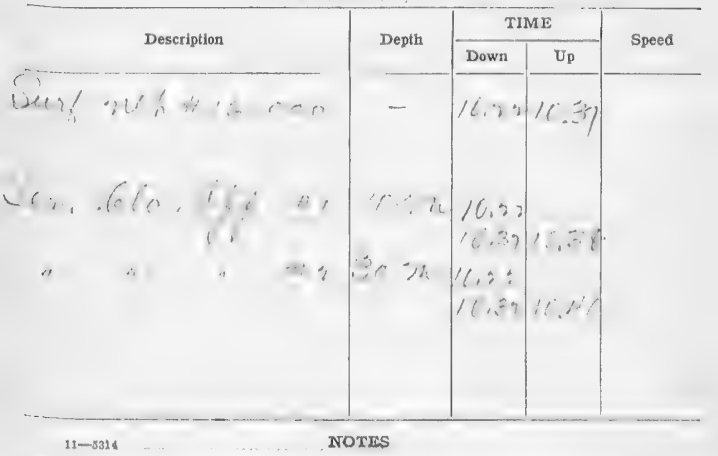
$\therefore t$

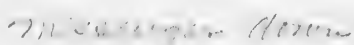
$-1 i^{2}=$
29
$\because \quad-1037$ C.LC 
Station No... If 6740 Date: fuly 261916 Hour: $/ 0$ inf 6 a.mo to /1.05 L.m.

Position:- Lat.

Long.

Locality:

Depth: $[3-2) \mathrm{V}$ Bottom:

Sample:

$$
24.72
$$

Wind: Direction,

Force,

Sea:

Sky:

TEMPERATURES

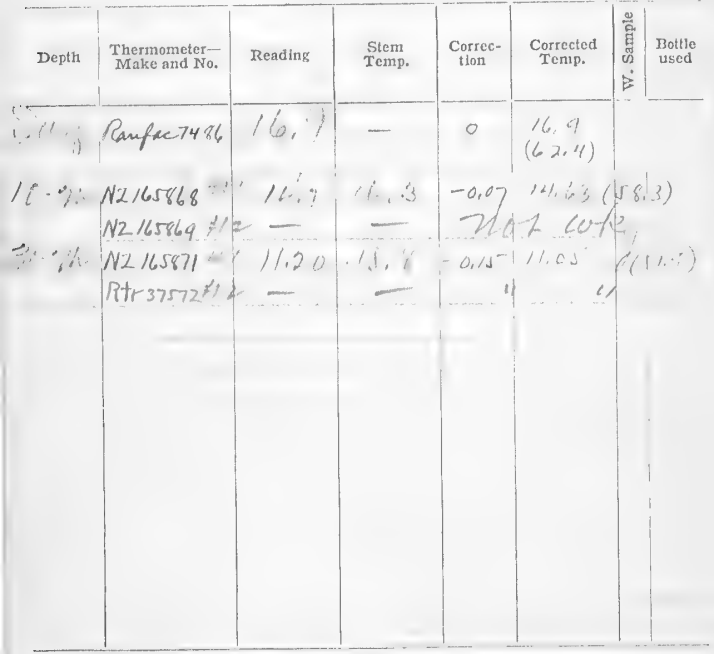

CURRENTS

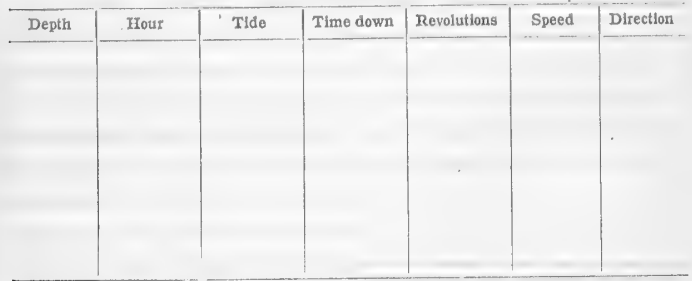

Color of water:

Transparency:

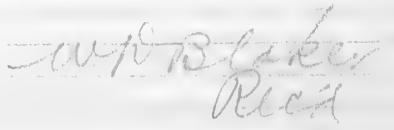




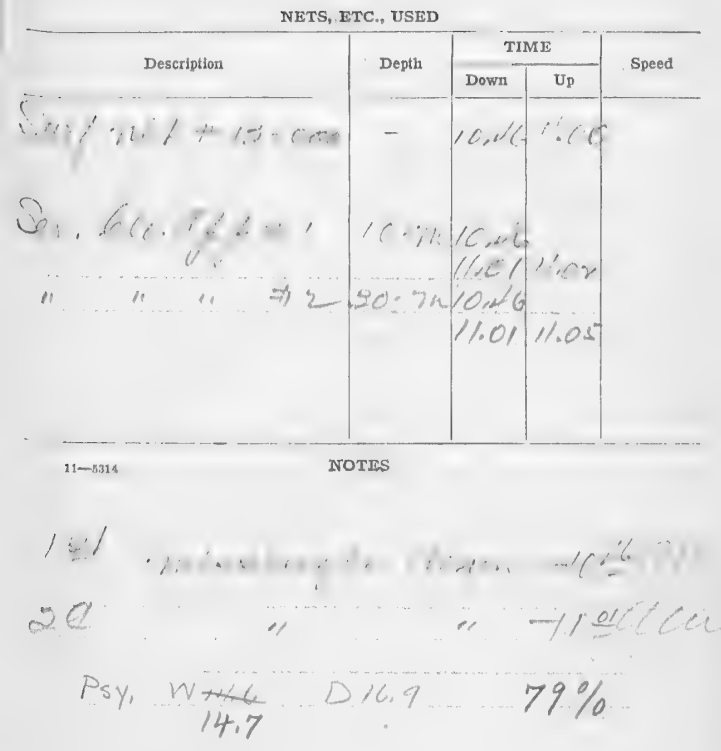

Chat 5200 C.F S.S.
1100 4.7n.; Right truzent San merias bease $n 711^{\circ}$ W (tue) divint it s.with 
Station No. $\because y$, Datc: $/$, Irour: $/, 120$. M. to $1=1$.M.

Position: Lat. Long.

Locality:

Depth: $5=\cdots /$ Bottom: Sample:

24 $2 \pi x$

Wind: Direction,

Force,

Sea:

Sky:

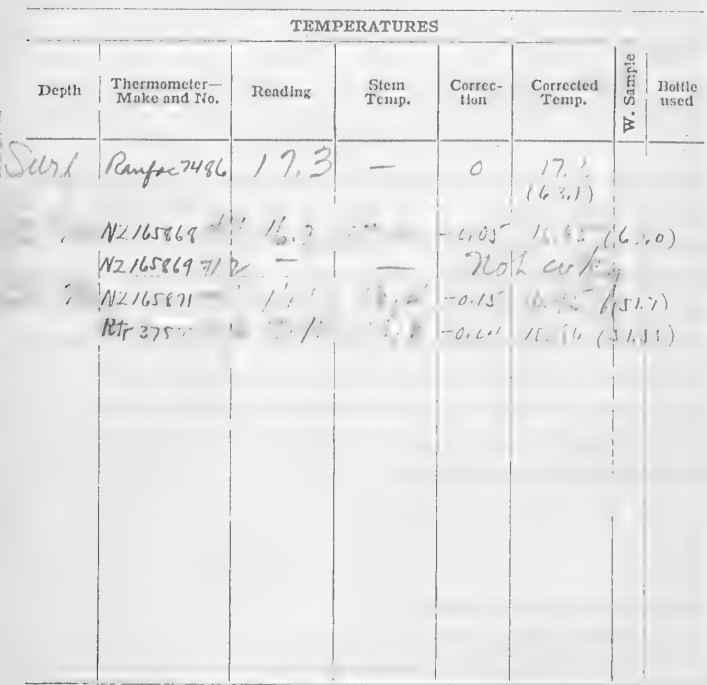

CURRENTS

\begin{tabular}{l|l|l|l|l|l|l}
\hline Depth & Hour & Tide & Time downt & Revolutions & Speed & Direction \\
\hline & & & & & \\
& & & & & \\
& & & & & \\
\end{tabular}


Station No. $-f /-671 \frac{1}{6}$ Date: $i \cdot 191$ Hour: .... Q.m.to d. . .

Position: Lat.

Long.

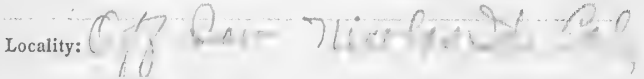

Depth: $=$ rit Bottom: $292 \mathrm{nw}$

Sample:

Wind: Direction,

Force,

Sea:

Sky:

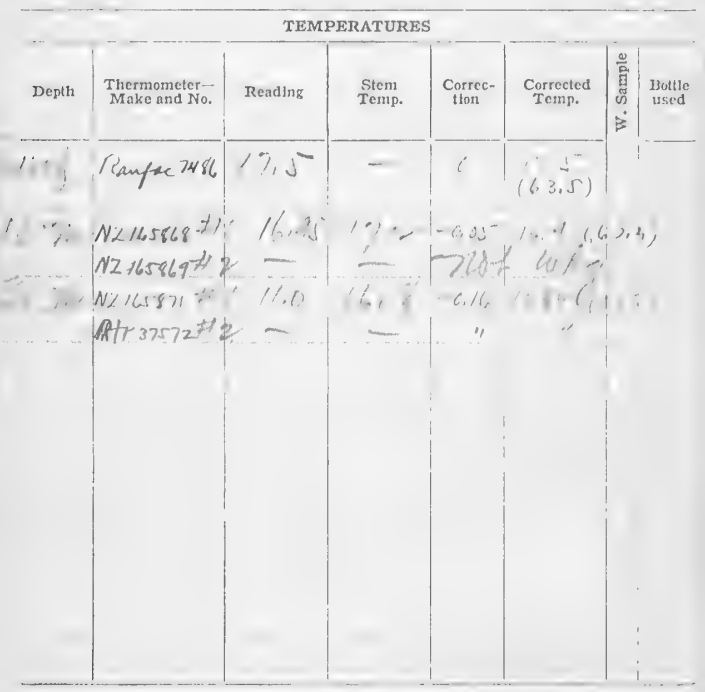

CURRENTS

\begin{tabular}{|c|c|c|c|c|c|c|}
\hline Depth & Hour & Tide & Time down & Revolutions & Speed & Direction \\
\hline & & & & & & \\
\hline & & & & & & \\
\hline & & & & & & \\
\hline & & & & & & \\
\hline & & & & & & \\
\hline
\end{tabular}

Color of water:

Transparency:

REMARKS:

$11-\$ 314$

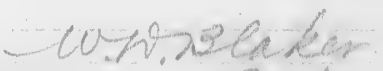


NETS, ETC., USED

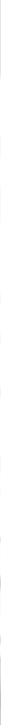




\section{NETS, ETC., USED}

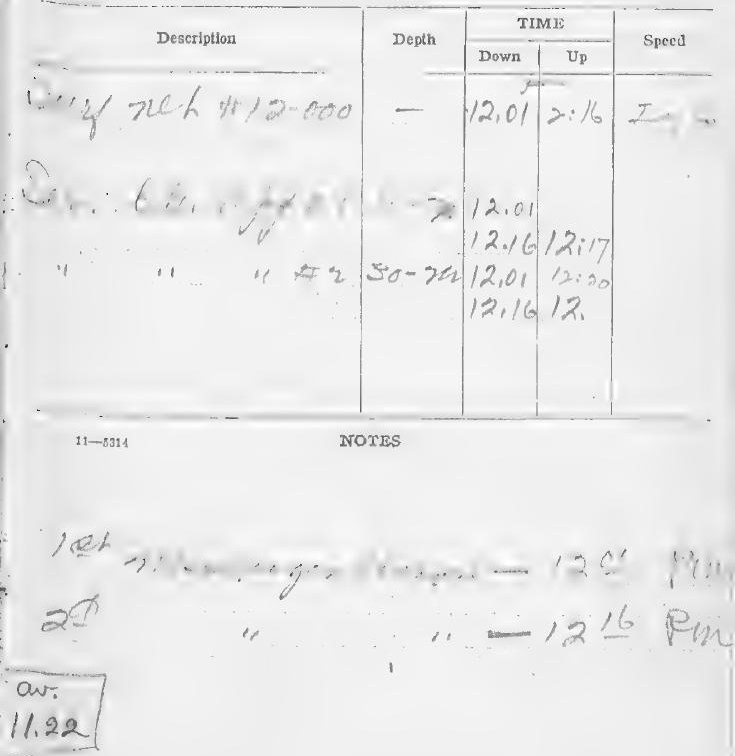


NETS, ITC., USED

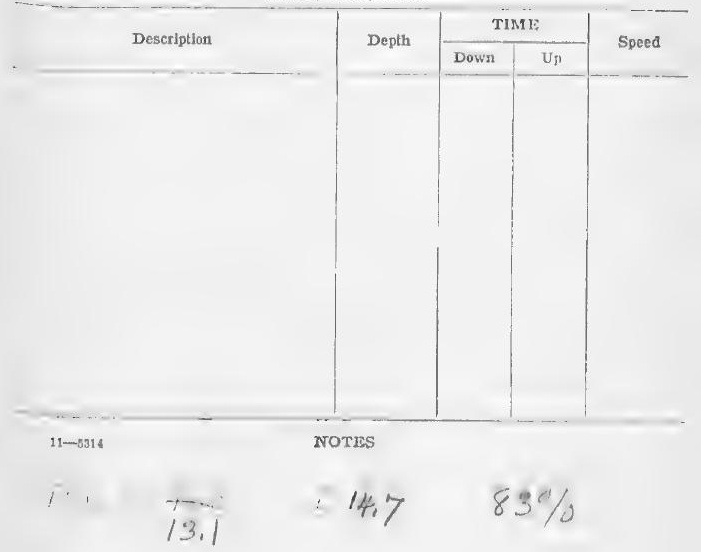

$$
\begin{gathered}
\text { Chant } 5200 \text { C.9 Y.S. } \\
\text { (imcherags) }
\end{gathered}
$$

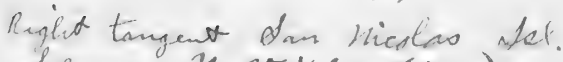
hears $4371^{\circ} \mathrm{W}$ (twe) dristant 1.8 miles

Lefst trangent dom thesins ata.

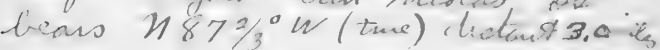


NETS, ETC, USED

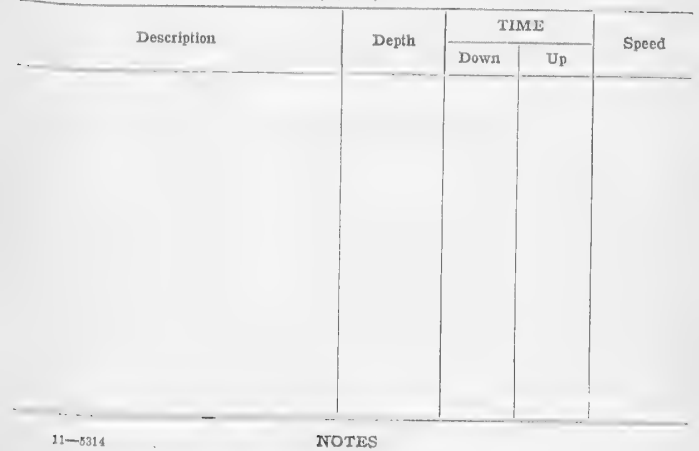

Chait 5802 C.9 S.S. 


$$
\text { Station Tro. Date: }(j) \dot{c}_{i} \text { is }
$$

Hour: $12 ; 00$. Muthigif .to . M.

Position: Lat. $33-10-24 . \mathrm{L}$ Long. $119-29-\because-2:$

\section{Locality:}

Depth:

Bottom:
Wind: Direction,

Sea: -..

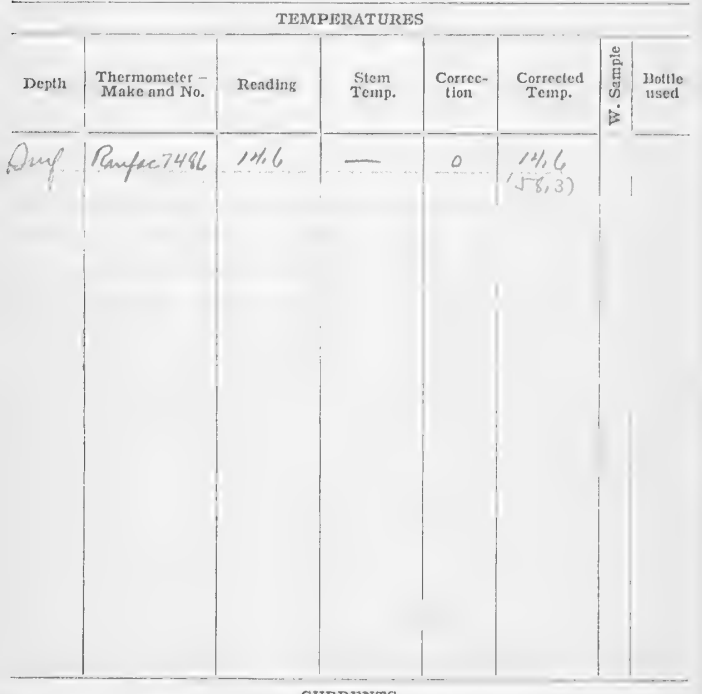

CURRENTS

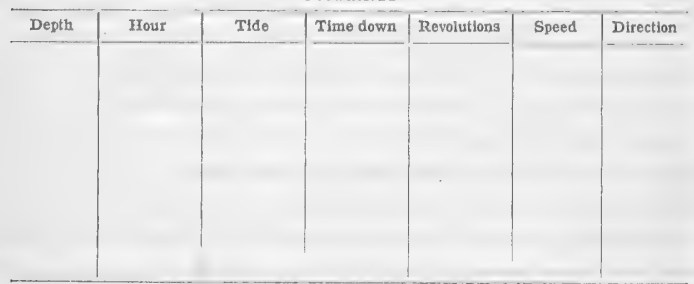

Color of water:

Transparency:
Force,

Sky: 
NETS, ETC., USED

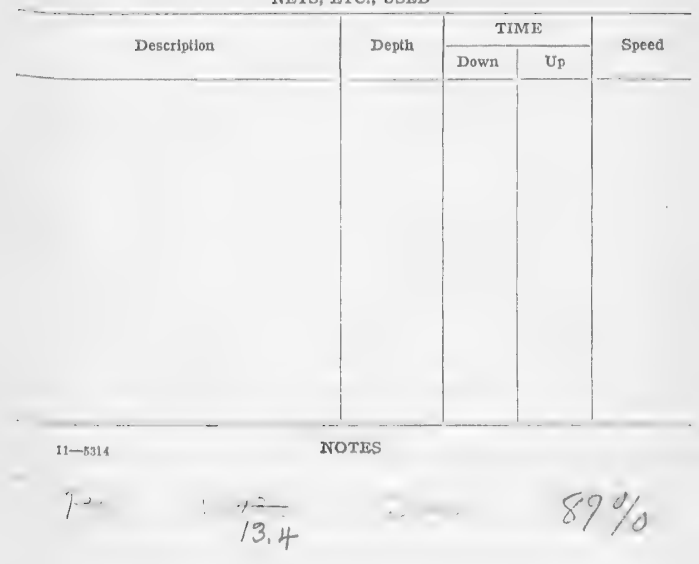

chut 5002 E'ES 
NETS, ETC., USTD

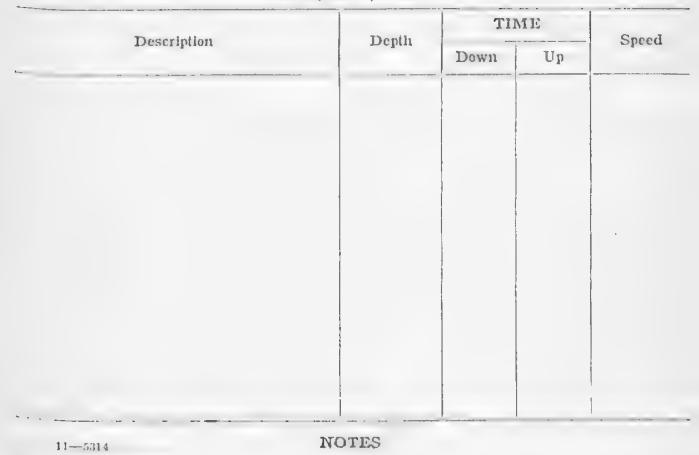

$11-5314$

NOTES

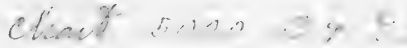


NETS, ETC, USED

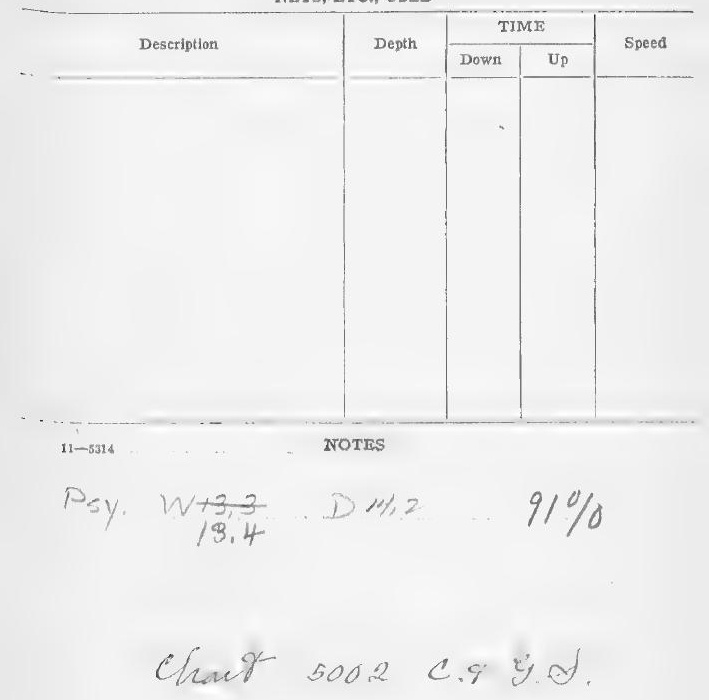


station No. $467 \% 6$

Date: $\bigcirc u h / 27 \ldots$.

Hour: $/: 30$ A.M. to .M.

Position: Lat. $33^{\circ}-10^{\prime}-12^{\prime \prime} \mathrm{N}$ Long. $119^{\circ}-39^{\prime}-544^{\circ}$ )

\section{Iocality:}

\section{Depth:}

Bottom:

Sample:

Wind: Direction,

Force,

Sea:

Sky:

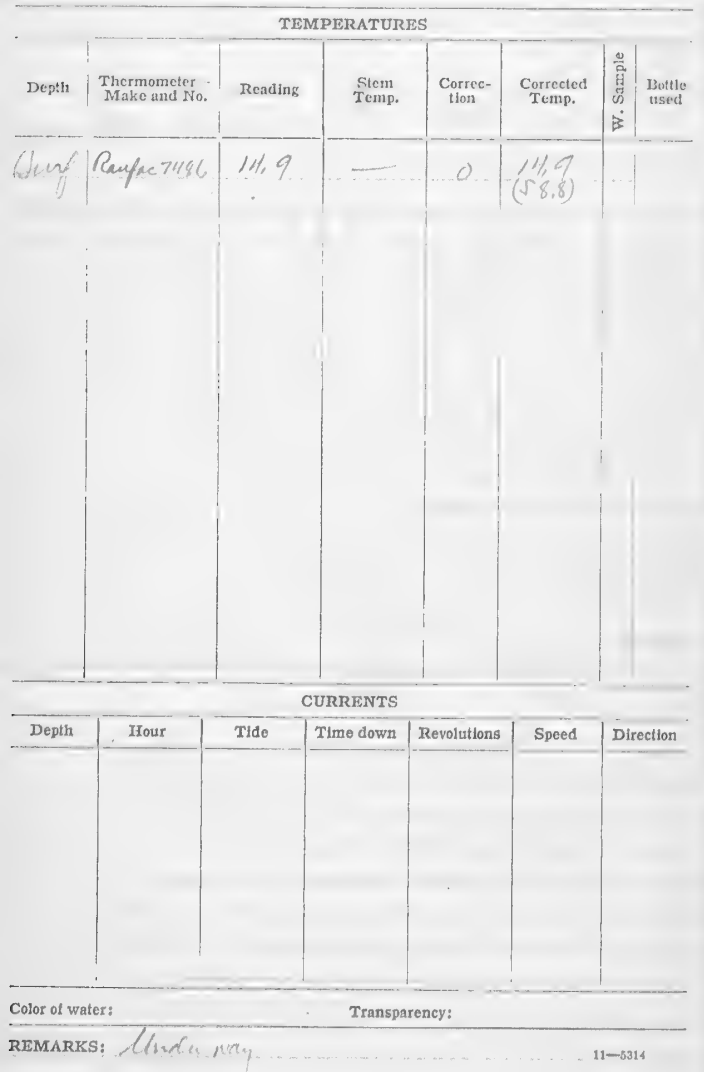


NETS, ETC., USED

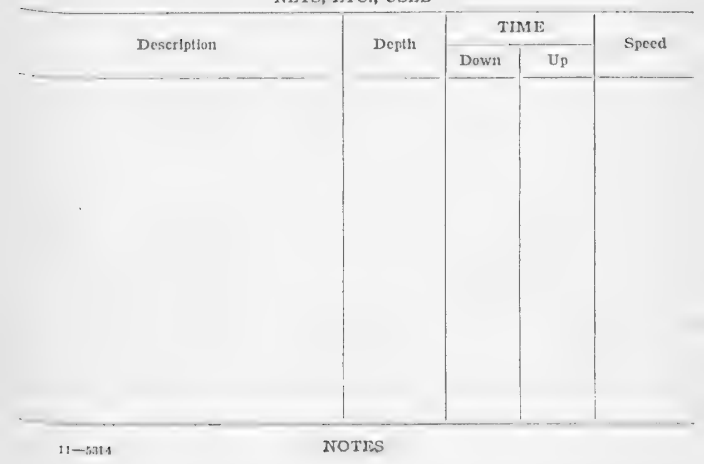

chen 5002 \&. $\% 5$ 


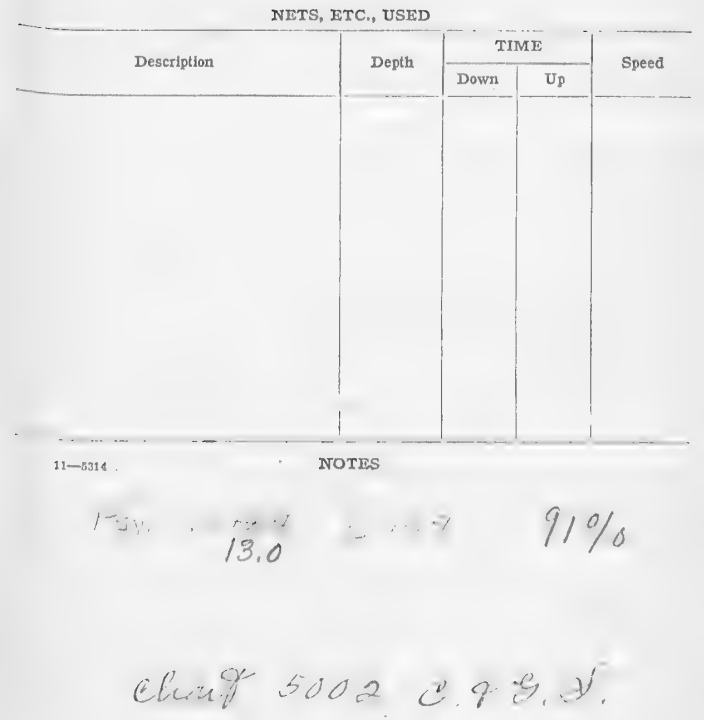


NETS, ETC., USED

Description $\quad$ Depth

$$
\text { chat } 5002 \text { C.7 I.S. }
$$


Station No. $H$ is ?

Date:

$101 / 1$

Hour: 3 ioo A.M. to

. M.

Position: Lat. 33-10-06 $M$ Long. $119^{\circ}-51-00^{\prime \prime}$,I

Locality:

Depth:

Bottom:

Sample:

Wind: Direction,

Force,

Sea:

Sky:

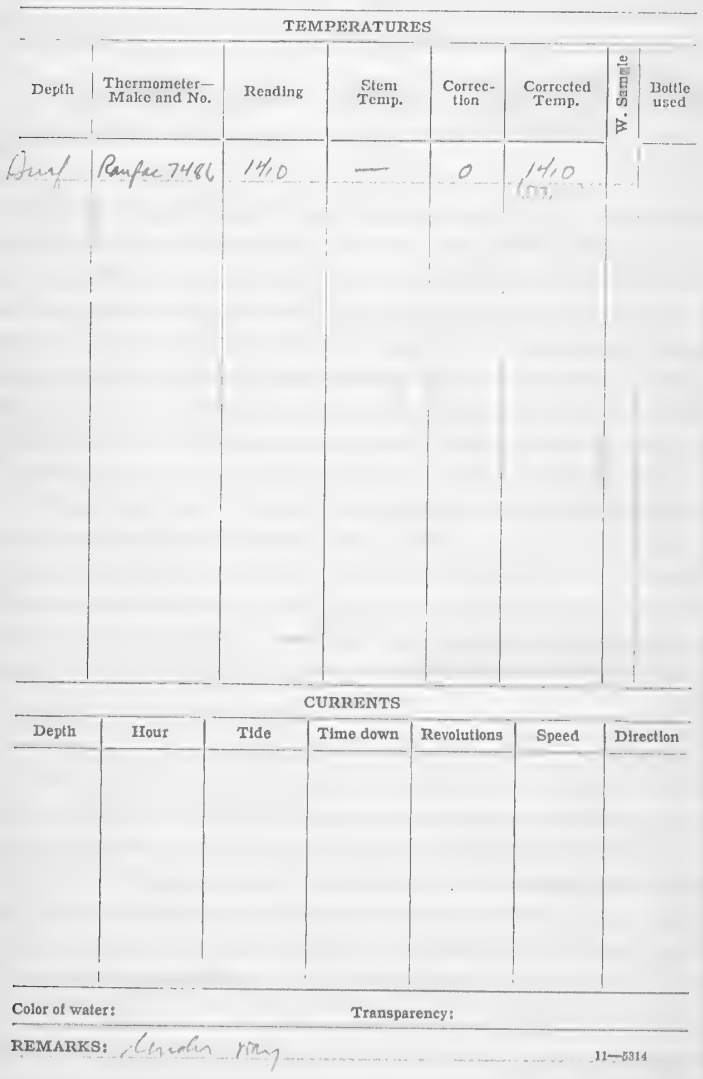




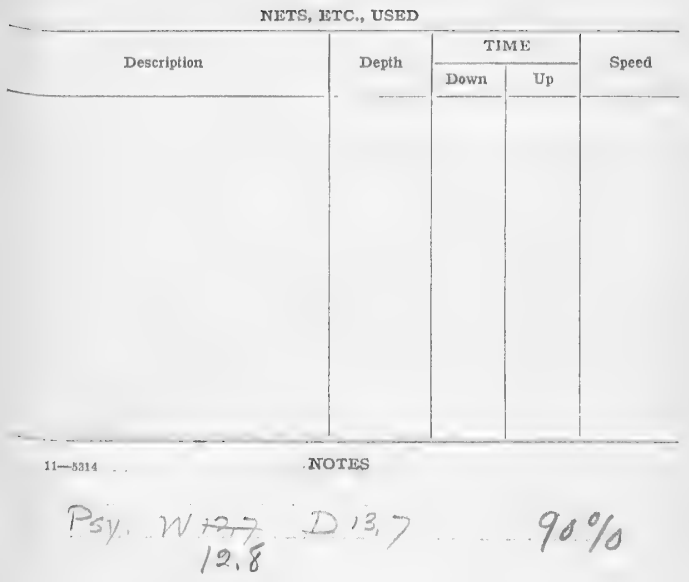

chaig 5002 C.F IS.S. 
Station No. $116>50$

Date: Ofub $>7 \ldots \ldots 101$

Hour: $3: 30 \% . M$, to -

. M.

Position: Lat. $33^{\circ}-10^{\circ}-00^{\prime \prime} \geqslant$ Long. $1 ! 1+\ldots \ldots \ldots$

Locality:

Depth:

Bottom:

Sample:

Wind: Direction,

Force,

Sea:

Sky:

-.

TEMPERATURES

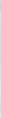

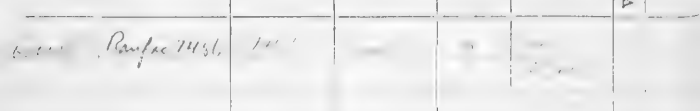
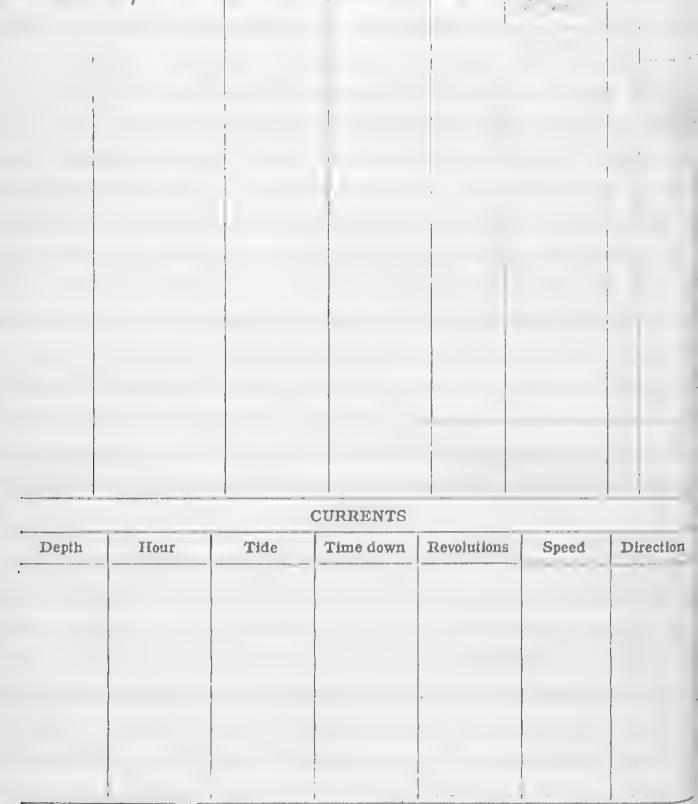

Color of water:

Transparency:

REMARKS: Llu Cu pran

11-5914 


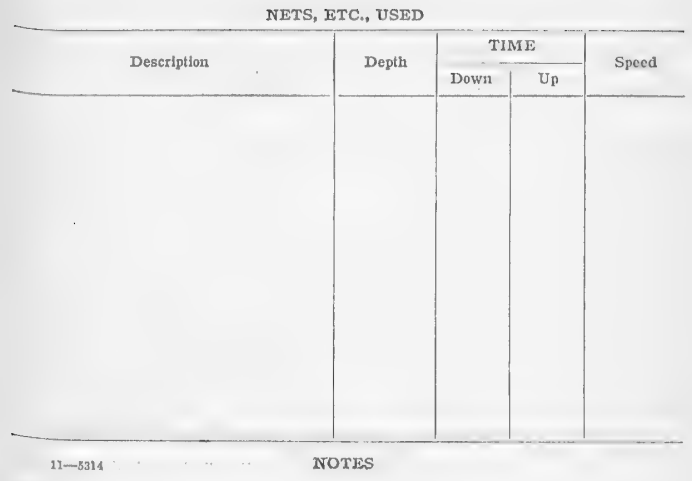

Chene $\therefore=\because \because$ 
Station No. H $6 \geqslant 51$

Date:

1916

Hour: 100 A. M. to .M.

Position: Lat. $33^{\circ}-10^{\prime}-00^{\prime \prime} \geqslant$ Long. $119-57-30^{\prime \prime}$

Locality:

Depth:

Bottom:

Sample:

Wind: Direction,

Force,

Sea:

Sky:

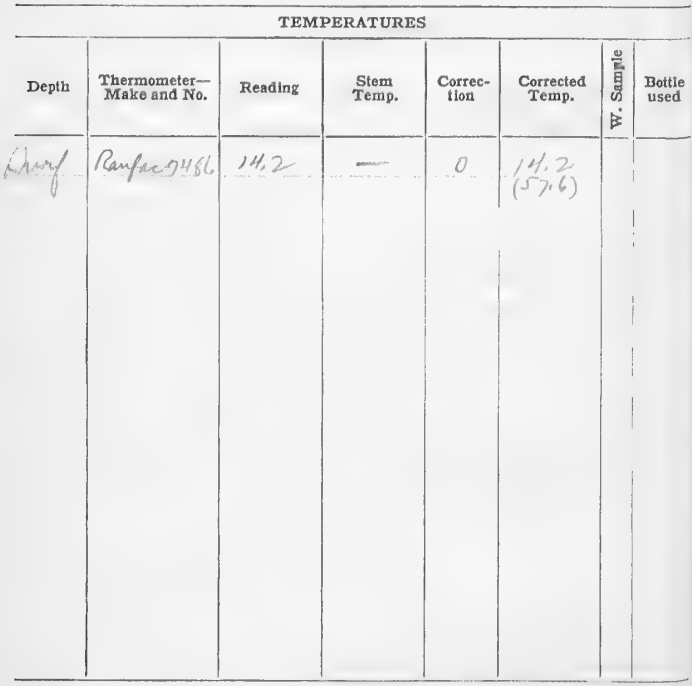

CURRENTS

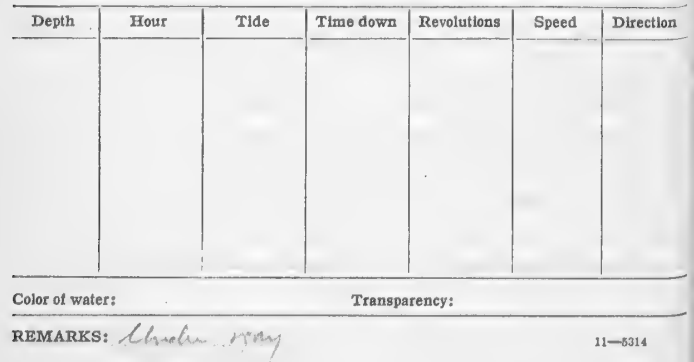


NETS, ETC., USED

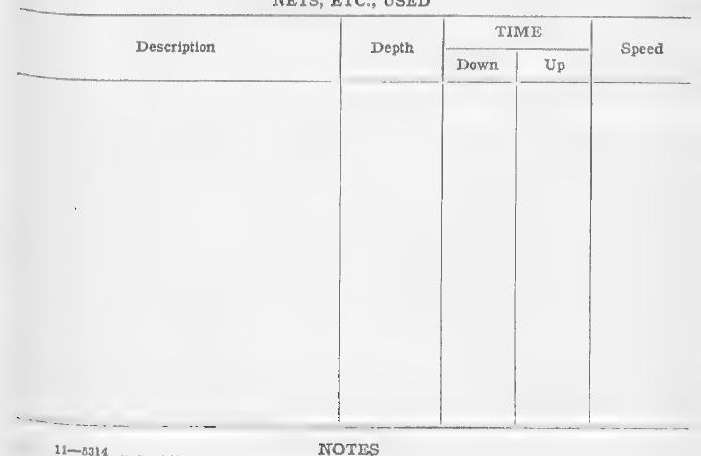

$11-5311$ NOTES

i=sy. $\quad \frac{w+2.8}{12.9} \quad D 13,7 \quad 91 \%$

Chaw 5002 C.F ES. 
Station No. $46 \% \sqrt{2}$

Date: Suly 2

Hour: $f: 30$ A.M. to $\quad . M$.

Position: Lat. $33-10^{\prime}-00^{\prime \prime} 2 /$ Long. $: ? .3$

Locality:

Depth:

Bottom:

Sample:

Wind: Direction,

Force,

Sea:

Sky:

TEMPERATURES

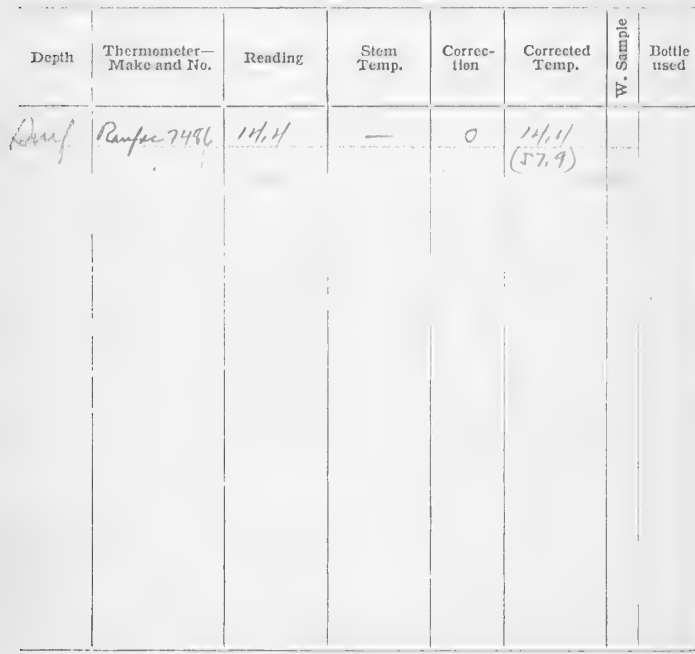

CURRENTS

\begin{tabular}{l|l|l|l|l|l|l}
\hline Depth & Tour & Tide & Time down & Revolutions & Speed & Direction \\
\hline & & & & & \\
& & & & & \\
& & & & & \\
\end{tabular}

Color of water:

Transparency:

REMARKS: LCuple nay 
NETS, TTC., USED

Description


Station No. $/ 16753$

Date: $\int 46,77 \ldots 1916$

Hour: $5: 00, A . M$. to $\ldots . . . M$.

Position: Lat. $33^{\circ}-10^{\prime}-00^{\prime \prime}$ \& $\quad$ Long. $120-01 \therefore . .$.

Locality:

Depth:

Bottom:

Sample:

Wind: Direction,

Force,

Sea:

Sky:

TEMPERATURES

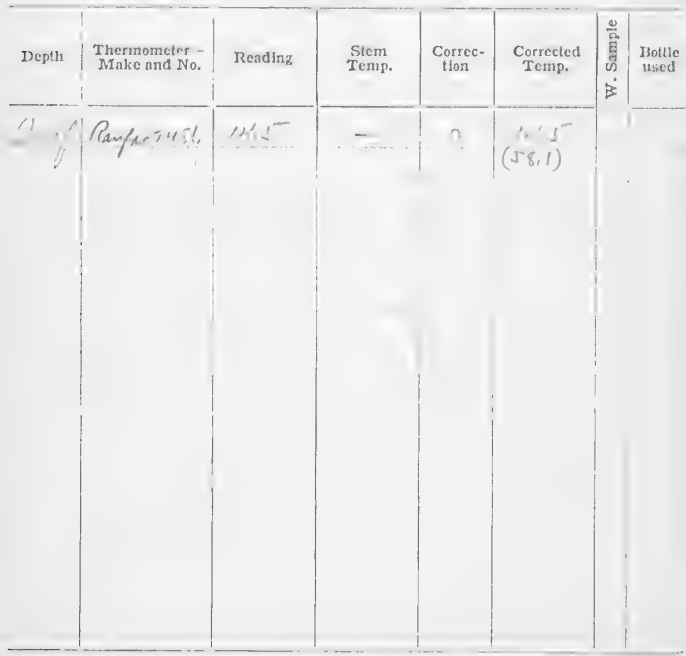

CURRENTS

\begin{tabular}{l|l|l|l|l|l|l}
\hline Depth & Hour & Tide & Time down & Revolutions & Speed & Direction \\
\hline & & & & & & \\
& & & & & & \\
& & & & & & \\
\hline Color of water: & & & & & \\
\hline REMarks: Climater & & & & & \\
\hline
\end{tabular}




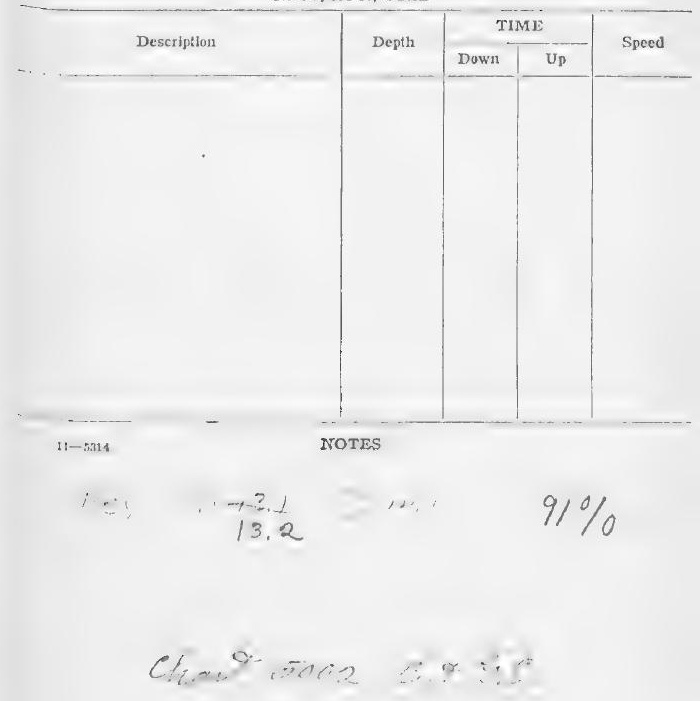


Station No. $/ 1 / \%$

Datc:

191(

Hour: $5: 30$ A.M.to $-\ldots$........

Position: Lat. $33^{\circ}-10^{\prime}-00^{\prime \prime} 21$ Long. $120-08-1$

\section{Locality:}
Depth:
Bottom:
Sample:

Wind: Direction,

Force,

Sea:

Sky:

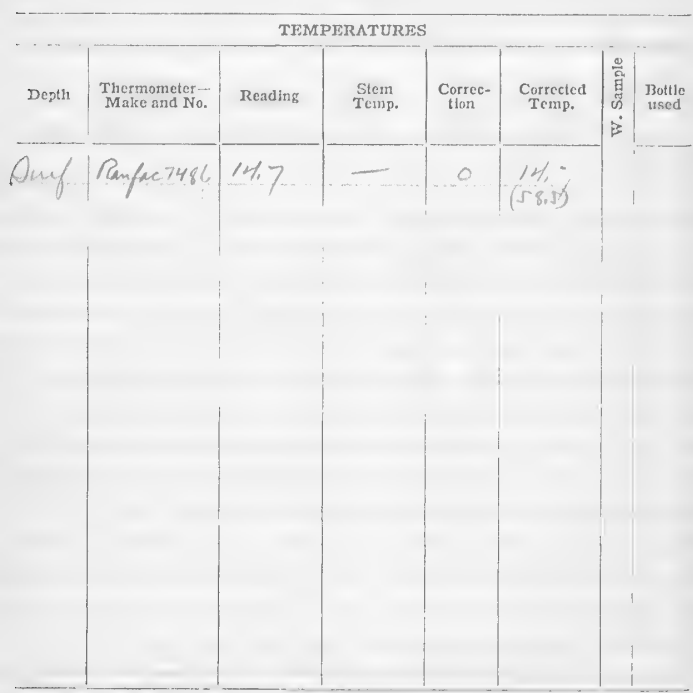

CURRENTS

\begin{tabular}{l|l|l|l|l|l|l}
\hline Depth & Hour & Tide & Time down & Revolutions & Speed & Direction \\
\hline & & & & \\
& & & & & \\
& & & & & & \\
& & & & & & \\
\hline
\end{tabular}

Color of water:

Transparency:

REMARKS:

clude mory 
NETS, ETC., USED

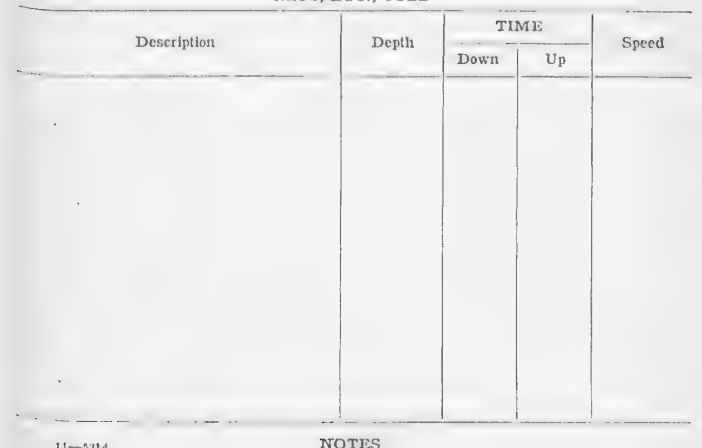

11-5:314

NOTES

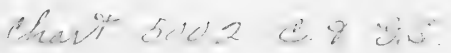


Station No. $t / l, i$

Hour: 6 ío A. M. to
Date:

$191 \zeta$

Position: Lat. $33-10-00$ 21 Long. :

Locality:

Depth:

Bottom:

Sample:

Wind: Direction,

Force,

Sea:

Sky:

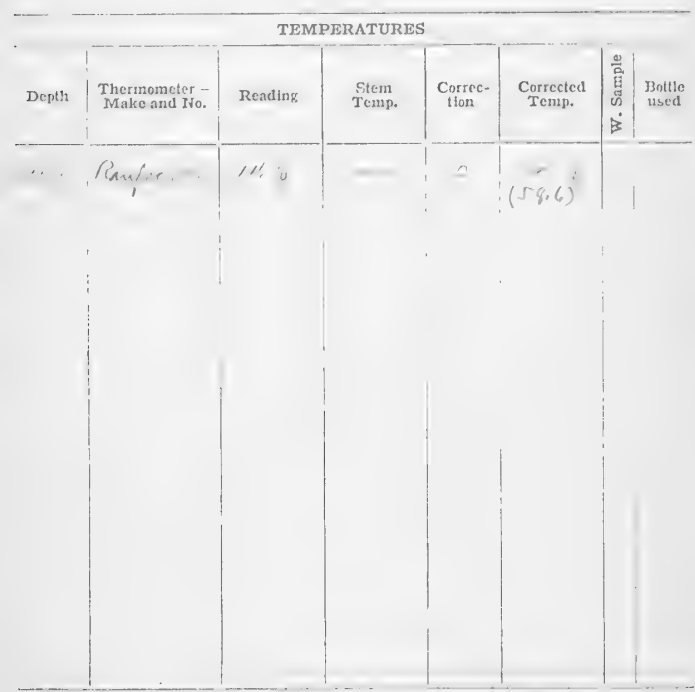

CURRENTS

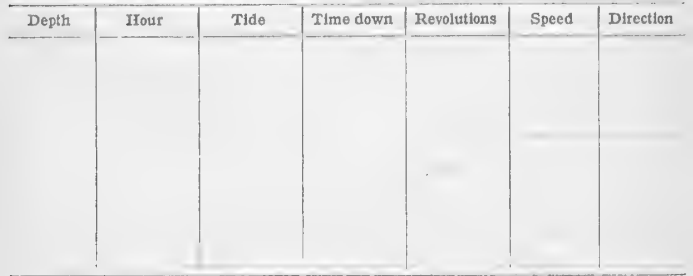

Color of water:

Transparency:

REMARKS:

- Chatum rome

y 
NITS, ETC., USED
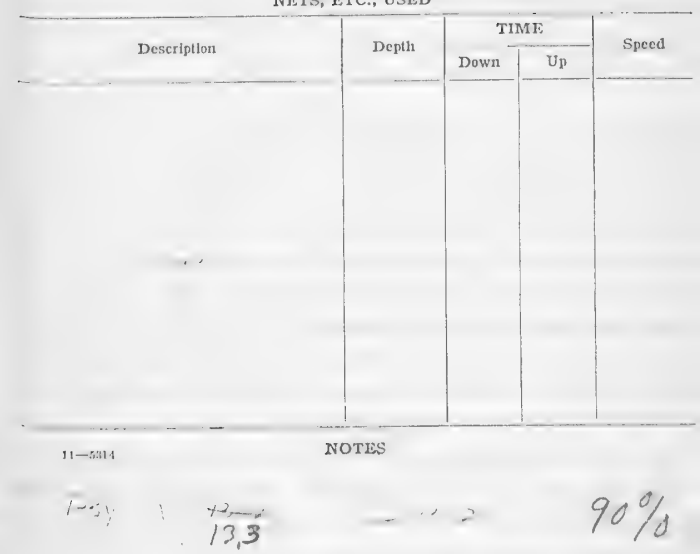

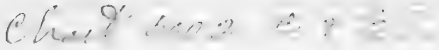


NETS, ETC., USED

Description

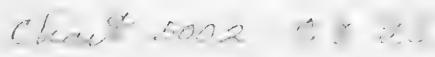


Station No. $/ 1, ; ! ?$

Date:

$191 /$

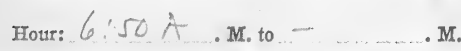

Position: Lat. $33^{\circ}-08^{\prime}-24^{\prime \prime} M$ Long. $120-10^{\prime}: \because$.

Locality:

Depth:

Bottom:

Sample:

Wind: Direction,

Force,

Sea:

Sky:

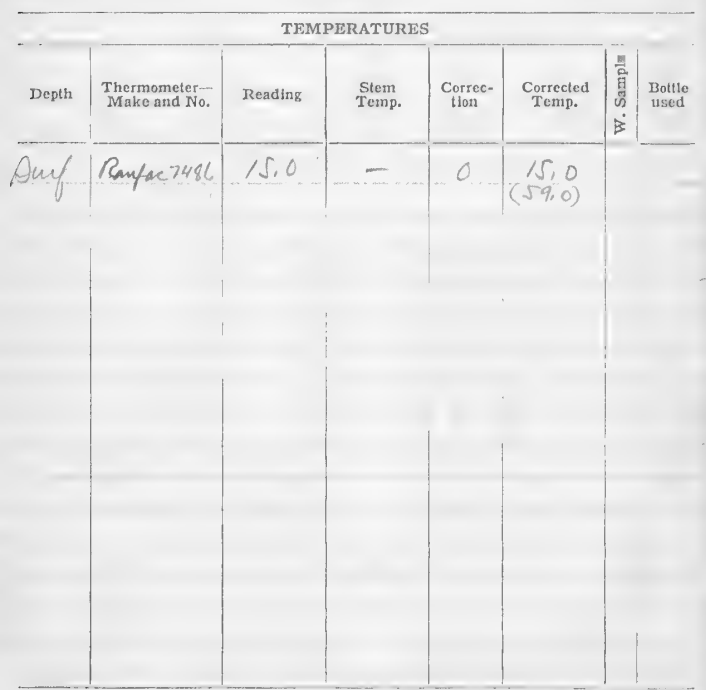

CURRENTS

\begin{tabular}{|c|c|c|c|c|c|c|}
\hline Depth & Hour & Tide & Time down & Revolutions & Speed & Direction \\
\hline & & & & & & \\
\hline & & & & & & \\
\hline & & & & & & \\
\hline & & & & & & \\
\hline & $=$ & & & & & \\
\hline & & & & & & \\
\hline
\end{tabular}


Station No. 41675.8

Date: Guly?

1916

Hour: $7: 30$ A. M. to $-\ldots \ldots$. M.

Position: Lat. $33^{\circ}-06^{\prime}-36^{\prime \prime} \geqslant$ Long. $120^{\circ}-07^{\prime}-00^{\prime \prime} \omega$

Locality:

Depth:

Bottom:

Sample:

Wind: Direction,

Force,

Sea:

Sky:

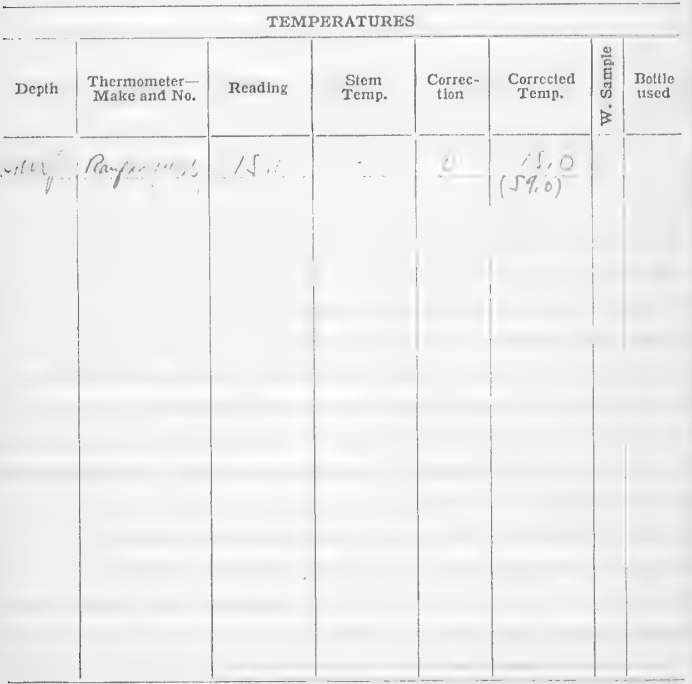

CURRENTS

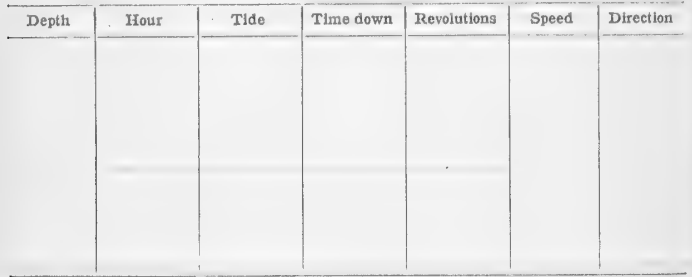

Color of water:

Transparency:

REMARKS: leuple wry 
Station No. 46759

Date: gul, 27

1916

Hour: 8; o o A.M. to . M.

Position: Lat. $33^{\circ}-04-42^{\prime \prime} 21$ Long. $12 \theta^{\circ} .03-30^{\prime \prime}$ " Locality:

Depth:

Bottom:

Sample:

Wind: Direction,

Sea:

-

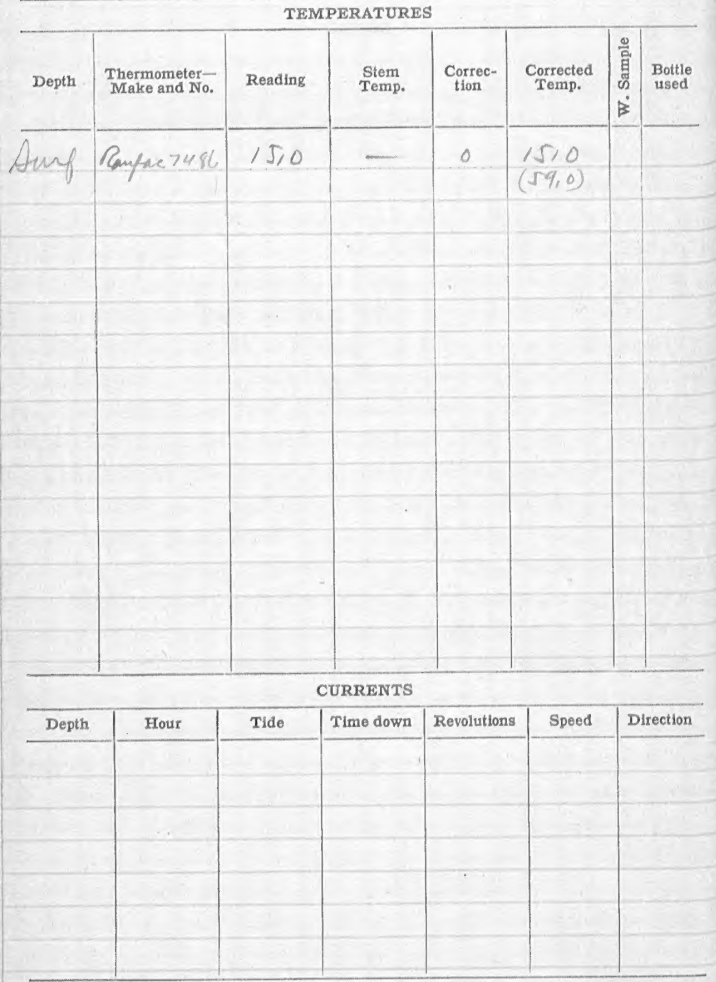

Force,

Sky:

REMARKS: Cardu nam … 11-5814




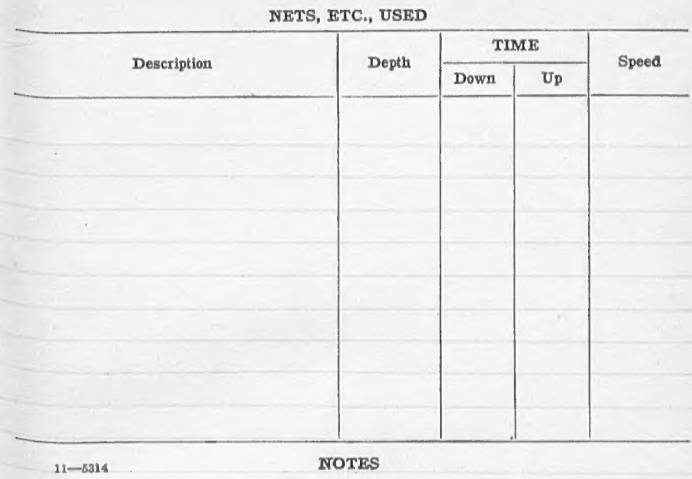

$11-6314$

NOTES

Psy. W $\frac{3.6}{13.9}$

$016.079 \%$

chatt 5002 C.4 I.S. 


$$
\begin{aligned}
& 26 \\
& 15 \\
& 125 \\
& \frac{20}{37.65} \\
& 0.212 \\
& 157 / 2 \\
& 75.0 \\
& 9.375
\end{aligned}
$$

